\title{
UNBOUNDED COVERINGS OF RIEMANN SURFACES AND EXTENSIONS OF RINGS OF MEROMORPHIC FUNCTIONS
}

\author{
BY \\ HELMUT RÖHRL(1,2)
}

In two papers [6;7], Hurwitz dealt with unbounded coverings of compact Riemann surfaces $Y$. He was able to determine the number of ("geometrically") different coverings in case all resp. all but one ramified points of $Y$ split in a single point of order two and points of order one. In the present paper we take up this question and ask for upper and lower bounds of the number of different unbounded coverings of $Y$ which have a prescribed ramification type. In case the degree of the covering (= number of sheets) equals $n$ we find a lower bound that is very roughly $(n !)^{2 g+r-3}$ and an upper bound that is very roughly $(n !)^{2 g+r-1}$ where $g$ is the genus of the compact Riemann surface $Y$ and $r$ is the number of ramified points of $Y$. The tools used in getting these estimates are some results on permutation groups like:

(i) Every element of the alternating group $A_{n}$ of $n$ elements is a commutator.

(ii) Let $n \neq 4$ and let $\alpha$ be an element of the symmetric group $S_{n}$ of $n$ elements that is not the identity. If $\alpha \in A_{n}\left(\notin A_{n}\right)$, then every element of $A_{n}\left(S_{n}\right)$ can be written as a product of at most $n$ conjugates of $\alpha$.

(iii) Given an element of $S_{n}$ that is not the identity. Then there is another element of $S_{n}$ which, together with the first one, generates $S_{n}$.

The second part of the paper deals with extensions of algebraic function fields of one variable whose local splitting is prescribed. It is clear that nonisomorphic field extensions correspond to different unbounded coverings, but different unbounded coverings may belong to isomorphic field extensions. Therefore, an upper bound for the number of different unbounded coverings (with prescribed ramification type) is also an upper bound for the number of nonisomorphic field extensions (with prescribed local splitting). Surprisingly, it turns out that the above mentioned lower bound (concerning coverings) also serves in general as a lower bound for the number of nonisomorphic field extensions. This result is of some interest in connection with a potential class field theory of arbitrary ramified field extensions. In the third part, we determine completely the algebraic

Received by the editors August 1, 1961 and, in revised form, April 10, 1962.

(1) This research was supported by the United States Air Force through the Air Force Office of Scientific Research.

(2) The author is grateful to the referee for several suggestions. 
structure of those extensions of the ring $I(Y)$ of all holomorphic functions on a noncompact Riemann surface $Y$ which are defined by an unbounded covering of $Y$ of finite degree; those extensions are characterized by being integrally closed, having no zero divisors, and being free $I(Y)$-modules of finite rank.

1. Some result on permutation groups. Let $E$ be a set and denote by $S_{E}$ the set of bijective mappings of $E$ onto itself equipped with the usual composition of mappings. $S_{E}$ is called the symmetric group of $E$. In case $E$ is a finite set consisting of $n$ elements we write also $S_{n}$ instead of $S_{E}$. Every permutation $\alpha$ of $E$, i.e., element of $S_{E}$, determines a partition $\pi(\alpha)$ of $E$ whose elements are the orbits of the cyclic group generated by $\alpha$. We order the elements of an orbit as follows: $\left(\cdots, \alpha(x), x, \alpha^{-1}(x), \cdots\right)$, provided the orbit is infinite, and $\left(\alpha^{n-1}(x), \cdots, x\right)$, provided the orbit contains exactly $n$ elements. These objects may be viewed as injective mappings of the ordered set of integers resp. the ordered set $(1,2, \cdots, n)$ into $E$. After having identified two mappings of $(1,2, \cdots, n)$ into $E$ if and only if one is the composition of the other one and a cyclic permutation of $(1,2, \cdots, n)$, we speak of cycles in $E$. Then each element of $S_{E}$ determines a set of mutually disjoint cycles in $E$ such that every element of $E$ is contained in some cycle. This establishes a bijective correspondence between $S_{E}$ and the family of such sets of cycles. Defining for two such sets of cycles the product in the obvious way we get a group that is isomorphic to $S_{E}$ and which also shall be denoted by $S_{E}$.

Given a partition $\pi$ of $E$ we denote by $q_{k}(\pi), k=1,2, \cdots, \aleph_{0}$, the cardinality of the set of those elements of $\pi$ which contain exactly $k$ elements. Later on we have to use frequently the well-known fact that two permutations $\alpha$ and $\beta$ are conjugate to each other if and only if for every $k$ the equation $q_{k}(\pi(\alpha))=q_{k}(\pi(\beta))$ holds. Denoting by $N(\pi)$ (or $N\left(q_{1}(\pi), q_{2}(\pi), \cdots\right)$ the cardinality for the set of all permutations $\alpha$ of $E$ for which $q_{k}(\pi(\alpha))=q_{k}(\pi)$ holds for every $k$, we get

Proposition 1.1. If $\operatorname{card} E=n<\aleph_{0}$, then

$$
N\left(q_{1}, q_{2}, \cdots\right)=\frac{n !}{\prod\left\{k^{q_{k}} \cdot q_{k} ! \mid k=1,2, \cdots\right\}} .
$$

If $\operatorname{card} E=\aleph_{0}$, then $N\left(q_{1}, q_{2}, \cdots\right)$ equals 1 resp. $\aleph_{0}$ depending on whether

$$
\Sigma\left\{q_{k} \mid k=1,2, \cdots, \aleph_{0}\right\}=0 \text { or } \neq 0 .
$$

Proof. The case card $E=\aleph_{0}$ is obvious. For the case $\operatorname{card} E=n<\aleph_{0}$ an elementary proof can be found in [14].

Proposition 1.2. Let $E$ be any set. Then every element of the commutator subgroup $\left[S_{E}, S_{E}\right]$ of $S_{E}$ is already a commutator. In case card $E \geqq \aleph_{0}$ we have in addition $\left[S_{E}, S_{E}\right]=S_{E}$.

Proof. The following formulas can be checked easily:

If $m$ is even: 


$$
\begin{aligned}
\{(12)(34) \cdots(m-1, m)\} \cdot\{(23)(45) \cdots(m-2, m-1)\} & (m, m, m-2, \cdots, 4,2) \\
= & (1,3,5, \cdots, m-1, m, m-2, \\
\{(23)(45) \cdots(m-2), m-1)\} \cdot\{(12) & (34) \cdots(m-1, m)\} \\
= & (2,4,6, \cdots, m-2, m, m-1, \cdots, 3,1) ;
\end{aligned}
$$

if $m$ is odd:

$$
\begin{aligned}
\{(12)(34) \cdots(m-2, m-1)\} \cdot\{(23) & (45) \cdots(m-1, m)\} \\
& =(1,3,5, \cdots, m, m-1, m-3, \cdots, 4,2)
\end{aligned}
$$

and finally

$$
\{(12)(34)(56) \cdots\} \cdot\{(23)(45)(67) \cdots\}=(\cdots, 8,6,4,2,1,3,5,7, \cdots) .
$$

(a) Suppose card $E=n<\aleph_{0}$. Then $\left[S_{E}, S_{E}\right]$ is the alternating group $A_{n}$. An element of $S_{n}$ belongs to $A_{n}$ if and only if it can be written as product of an arbitrary number of cycles of odd lengths and an even number of cycles of even lengths, all of which are mutually disjoint. Using (4) for the cycles of odd length and the two formulas (3) alternately for the cycles of even length we see that the given element in $A_{n}$ can be written as a product of two permutations, each of which is the product of the same number of mutually disjoint transpositions. Two such permutations are conjugate to each other. In addition a permutation of this last type equals its inverse. Therefore, the given element is a commutator.

(b) Suppose card $E \geqq \aleph_{0}$. Again every element of $S_{E}$ is the product of (possibly infinitely many) mutually disjoint cycles. If at least one of these cycles is infinite or else if there are infinitely many cycles of length $\geqq 2$, then we apply to each cycle (3), (4), or (5) respectively and show as in (a) that this product is a commutator. If the element in discussion is the product of only finitely many finite cycles, we proceed like that: the cycles of odd lengths are taken care of by (4), while pairs of cycles of even lengths are being treated by (3); so it remains to be shown that a single cycle of even length is a commutator. Without loss of generality we may restrict ourselves to the case $E=\{1,2,3, \cdots\}$ and the cycle $(1,2, \cdots, 2 k)$. Denoting $(2 k+1, \cdots, 4 k)(4 k+1, \cdots, 6 k) \cdots$ by $\beta$ we see that $\beta$ and $(1, \cdots, 2 k) \beta$ are conjugate which shows again that $(1, \cdots, 2 k)$ is a commutator.

Proposition 1.3. Every element in $A_{n}$ can be written as a commutator in at most $n ! p(n)\left({ }^{3}\right)$ different ways; the minimal number $q(n)$ of ways in which an arbitrary element of $A_{n}$ can be written as a commutator fulfills the inequality ${ }^{(4)}$

$$
q(n) \geqq\left[\frac{1}{2}(n+1-\sqrt{ }(n+1))\right] !\left(n-2\left[\frac{1}{2}(n+1-\sqrt{ }(n+1))\right]\right) ! 2^{\left[\left(n+1-(n+1)^{1 / 2}\right) / 2\right]} .
$$

Proof. $\sigma \tau \sigma^{-1} \tau^{-1}=\alpha$ is equivalent to the statement: $\tau$ is conjugate to $\alpha \tau$. Now suppose $\tau$ is conjugate to $\alpha \tau$. Then it is easy to see that the number of $\sigma$ 's for which $\sigma \tau \sigma^{-1}=\alpha \tau$ holds, equals

(3) $p(n)$ denotes the number of partitions of $n$ in positive integers.

(4) $[a]$ denotes the biggest integer $\leqq a$. 


$$
\prod\left\{k^{q_{k}(\tau)} \cdot q_{k}(\tau) ! \mid k=1,2, \cdots\right\} .
$$

Hence the number of times $\alpha$ can be written as commutator equals

$$
\Sigma\left\{\prod\left\{k^{q_{k}(\tau)} \cdot q_{k}(\tau) ! \mid k=1,2, \cdots\right\} \mid \tau \text { conjugate to } \alpha \tau\right\} .
$$

This number is not greater than

$$
\begin{aligned}
& \Sigma\left\{\prod\left\{k^{q_{k}(\tau)} \cdot q_{k}(\tau) ! \mid k=1,2, \cdots\right\} \mid \tau \in S_{n}\right\} \\
& \quad=\Sigma\left\{N\left(q_{1}, q_{2}, \cdots\right) \cdot \prod\left\{k^{q_{k}} \cdot q_{k} ! \mid k=1,2, \cdots\right\} \mid q_{1}+2 q_{2}+\cdots=n\right\}=n ! p(n),
\end{aligned}
$$

according to Proposition 1.1. Obviously, this number is reached in case $\alpha=1$. In order to get a lower bound, we proceed as follows. We write the permutation $\alpha$ as a product of mutually disjoint cycles. Assume that there are exactly $k$ cycles of odd lengths $\kappa_{1}, \cdots, \kappa_{k}$ respectively, $2 l$ cycles of even lengths $\lambda_{1}, \cdots, \lambda_{2 l}$ respectively, and $m$ fixed elements. Thus we have $n=\kappa_{1}+\cdots+\kappa_{k}+\lambda_{1}+\cdots+\lambda_{2 l}+m$. A repeated application of the formulas (3) and (4) shows that $\alpha$ can be written as $\tau^{\prime} \tau^{\prime \prime}$ where $\tau^{\prime}$ as well as $\tau^{\prime \prime}$ is the product of

$$
\frac{1}{2}\left(\kappa_{1}+\cdots+\kappa_{k}+\lambda_{1}+\cdots+\lambda_{2 l}-k-2 l\right)=\frac{1}{2}(n-k-2 l-m)
$$

mutually disjoint transpositions. Hence $\tau^{\prime}=\sigma \tau^{\prime \prime} \sigma^{-1}$ and $\alpha=\sigma \tau^{\prime \prime} \sigma^{-1} \tau^{\prime \prime-1}$ for a suitable choice of $\sigma$. As shown above there are

$\prod\left\{k^{q_{k}\left(\tau^{\prime \prime}\right)} \cdot q_{k}\left(\tau^{\prime \prime}\right) ! \mid k=1,2, \cdots\right\}=(k+2 l+m) !\left(\frac{1}{2}(n-k-2 l-m)\right) ! 2^{(n-k-2 l-m) / 2}$

different $\sigma$ 's for which $\tau^{\prime}=\sigma \tau^{\prime \prime} \sigma^{-1}$ holds. In order to find a lower bound for $q(n)$ we consider the function $f(t)=(n-2 t) ! t ! 2^{t}$ where $0 \leqq t \leqq \frac{1}{2}(n-1)$. It is easy to check that the quotient $f(t) f^{-1}(t+1)$ is bigger than 1 for $0 \leqq t<\frac{1}{2}(n-\sqrt{ }(n+1))$ and smaller than 1 for $\frac{1}{2}(n-\sqrt{ }(n+1))<t \leqq \frac{1}{2}(n-1)$. Therefore $f(t)$ restricted to the integers in $0 \leqq t \leqq \frac{1}{2}(n-1)$ assumes its minimum for

$$
t_{0}=\left[\frac{1}{2}(n+1-\sqrt{ }(n+1))\right] .
$$

Proposition 1.4. Let $n \neq 4$ and let $\alpha$ be a permutation that is different from the identity. If $\alpha \in A_{n}\left(\alpha \notin A_{n}\right)$, then every element of $A_{n}\left(S_{n}\right)$ can be written as a product of at most $n$ conjugates of $\alpha$.

Proof. The statement can be checked immediately for $n=2$ and $n=3$. Hence we may assume $n \geqq 5$. Let $\alpha$ contain at least one cycle of length $\geqq 3$. Then there are two conjugates of $\alpha$ whose product is $(1,2,3)$ : If $\alpha$ contains a cycle of length 3 , say $(1,3,2)$, then replace the remaining cycles in $\alpha$ by their inverses; the product of this conjugate of $\alpha$ with $\alpha$ is evidently $(1,2,3)$. If $\alpha$ contains a cycle of length $k \geqq 4$, say $(1,2, \cdots, k)^{-1}$, then replace this cycle in $\alpha$ by $(1,3,2,4,5, \cdots, k)$ and the remaining cycles by their inverses; again the product of this conjugate of $\alpha$ with $\alpha$ itself is $(1,2,3)$ as to be seen from the formula

$$
(1,2,3)=(1,3,2,4,5, \cdots, k)(1,2,3, \cdots, k)^{-1} \text {. }
$$


Furthermore, we have

$$
(1,2,3)(1,4,5) \cdots(1,2 k, 2 k+1)=(1,2,3, \cdots, 2 k+1)
$$

and

$$
(1,2, \cdots, 2 k+1)(2 k+1,2 k+2, \cdots, 2 l)=(1,2, \cdots, 2 k)(2 k+1, \cdots, 2 l) .
$$

Now let $\beta \in A_{n}$ and write it as a product of mutually disjoint cycles. Assume that the cycles of odd length have lengths $\kappa_{1}, \cdots, \kappa_{k}$, while the cycles of even length have lengths $\lambda_{1}, \cdots, \lambda_{21}$. (6) and (7) show that $\beta$ can be written as product of at most

$$
\frac{1}{2}\left(\kappa_{1}+\cdots+\kappa_{k}+\lambda_{1}+\cdots+\lambda_{2 l}\right)
$$

conjugates of $(1,2,3)$. Because this number is obviously not greater that $n / 2$, we can now write $\beta$ as a product of not more than $n$ conjugates of $\alpha$. In case neither $\alpha$ nor $\beta$ is an element of $A_{n}$ we can find a suitable conjugate $\alpha^{\prime}$ of $\alpha$ so that $\alpha^{\prime-1} \beta \in A_{n}$ leaves at least one element fixed. Then, however, the corresponding sum (8) is smaller than $n$. The above argument implies that $\alpha^{\prime-1} \beta$ can be written as a product of less than $n$ conjugates of $\alpha$, which proves the statement for $\alpha$ 's subject to the restriction of the beginning of the proof.

Now let $\alpha=(1,2)(3,4) \cdots(2 k-1,2 k)$ and $2 k<n$. Then we have

$$
(1,2)(3,4) \cdots(2 k-3,2 k-2)(2 k-1,2 k+1)=(2 k-1,2 k, 2 k+1)
$$

and again the argument from before carries through.

If $n=2 k$ and $\alpha=(1,2)(3,4) \cdots(n-1, n)$, recall that the hypothesis $n \geqq 5$ in this case means $n \geqq 6$. It can be checked directly that the statement of Proposition 1.4 holds for $n=6$ and the above choice of $\alpha$. Hence we may assume $n \geqq 8$.

The formula $(1,2)(3,4)(1,3)(2,4)=(1,4)(2,3)$ shows that each product of an even number of disjoint transpositions is the product of two suitable conjugates of $\alpha$. Using again (3) and (4) we find that each element $\beta$ in $A_{n}$ can be written as the product of two permutations $\beta_{1}$ and $\beta_{2}$ each of which is the product of the same number of mutually disjoint transpositions. Hence $\beta$ is the product of at most 6 conjugates of $\alpha$ : if $\beta_{1}$ (as well as $\beta_{2}$ ) consists of an even number of mutually disjoint transpositions, then both $\beta_{1}$ and $\beta_{2}$ can be written as the product of at most two conjugates of $\alpha$, according to our last remark; if $\beta_{1}$ (as well as $\beta_{2}$ ) is the product of an odd number of mutually disjoint transpositions, then we pair a transposition of $\beta_{1}$ with a disjoint one of $\beta_{2}$ in order to write $\beta$ as a product of three permutations each of which is the product of an even number of mutually disjoint transpositions; applying our last argument once more we find that this $\beta$ can be written as product of at most 6 conjugates of $\alpha$. If neither $\alpha$ nor $\beta$ are in $A_{n}$, then $\alpha^{-1} \beta$ is in $A_{n}$ and can be written as product of at most 6 conjugates of $\alpha$. Hence $\beta$ itself can be written as product of at most 7 conjugates of $\alpha$. Because we have $n \geqq 8$ in this case, the required statement is proved. 
It may be remarked that Proposition 1.4 fails for $n=4$ : the element $(1,2)(3,4)$ and its conjugates are the only ones for which the statement does not hold.

Proposition 1.5. Let $\alpha \in S_{n}$ be a permutation which is different from the identity, and, in case $n=4$, is not conjugate to $(1,2)(3,4)$. Let $H$ be any transitive subgroup of $S_{n}$. Then there is an element $\beta$ that is the product of not more than $[(n+3) / 2]$ conjugates of $\alpha$ such that the subgroup $\langle H, \beta\rangle$ generated by $H$ and $\beta$ is primitive.

Proof. There is nothing to prove for $n=2$ and $n=3$. The case $n=4$ can be checked immediately. Therefore we may assume $n \geqq 5$. Suppose that $\alpha$ contains at least one cycle of length $\geqq 3$. If $n \equiv 1(4)$, then the cycle $\beta=(1, \cdots,(n+1) / 2)$ is the product of at most $(n-1) / 2$ conjugates of $\alpha$ as to be taken from (6). If $\langle H, \beta\rangle$ were not primitive, let $M$ be a domain of impritivity. $M$ cannot contain a digit of $\beta$ as well as a digit not appearing in $\beta$. because then $M$ would have to contain all digits of $\beta$ which is impossible. Hence every domain of imprimitivity is either a subset of $\{1, \cdots,(n+1) / 2\}$ or else has no digits in common with this set. Thus $(n+1) / 2$ as well as $(n-1) / 2$ are multiples of the cardinality of $M$. The g.c.d. of these two numbers being 1 implies now that $M$ consists of only one element, that is $\langle(H, \beta)\rangle$ is primitive. In case $n \equiv 0(4)$ the cycle $\beta=(1, \cdots, n / 2+1)$ has the required property as to be seen similarly. If $n \equiv 2(4), \beta=(1, \cdots, n / 2+2)$ fulfills the requirements of the proposition. In case $n \equiv 3$ (4) we choose $\beta=(1, \cdots,(n+1) / 2)((n+2) / 2,(n+3) / 2)$ which is the product of not more than $(n+3) / 2$ conjugates of $\alpha$ as we see from (6) and (7). Again a domain of imprimitivity $M$ cannot contain a digit $\leqq(n+1) / 2$ as well as a digit $>(n+3) / 2$. Moreover $M$ cannot contain the digits $(n+2) / 2,(n+3) / 2$ together with a digit $\leqq(n+1) / 2$ : in this case $M$ would consist of all digits $\leqq$ $(n+3) / 2$ which is impossible. However, if $M$ contains only one of the digits $(n+2) / 2$ and $(n+3) / 2$ and some digit $\leqq(n+1) / 2$, then $M$ contains exactly $((n+1) / 4)+1$ digits $\leqq(n+3) / 2$; therefore there are two domains of imprimitivity that cover the set $\{1, \cdots,(n+3) / 2\}$ which implies that $((n+1) / 2)+1$ has to be a divisor of $(n-3) / 2(=$ the number of digits $>(n+3) / 2)$. But this is impossible except for $n=11$ in which case nothing has to be proved anyway. Consequently, every domain of imprimitivity is either contained in the set $\{1, \cdots,(n+1) / 2\}$ or has no point in common with this set. From this we derive a contradiction in a similar way as before. Finally, we have to discuss the case where $\alpha$ is the product of mutually disjoint cycles of length 2 . If $\alpha=(1,2) \cdots(2 k-1,2 k)$ and $2 k<n$, we use one of the formulas in the proof of Proposition 1.4 in order to get the desired result as before. Suppose now $2 k=n$. If $n=8 l+2$ resp. $8 l+6$, then $\beta=\{(1,2)(3,4) \cdots(4 l+3,4 l+4)\}$. $\{(2,3)(4,5) \cdots(4 l+4,4 l+5)\}$ is a cycle of length $4 l+5$ that according to the proof of Proposition 1.4 is the product of 4 conjugates of $\alpha$. Obviously, it fulfills the requirements of our proposition. In case $n \equiv 0(8)$ the cycle 
$\beta=\{(1,2)(3,4) \cdots(n / 2-1, n / 2)\} \cdot\{(2,3)(4,5) \cdots(n / 2, n / 2+1)\}$ has length $n / 2+1$, is the product of 4 conjugates of $\alpha$ and fulfills the claim of the proposition. In case $n \equiv 4(8)$ every element of $S_{n}$ is the product of not more than 6 conjugates of $\alpha$ as was shown in the proof of Proposition 1.4. Hence an appropriate element $\beta$ in $S_{n}$ can be found which fulfills the statement.

It may be remarked, that in case $n=4$ and $\alpha$ is not conjugate to $(1,2)(3,4)$ we need only the product of at most two conjugates of $\alpha$ in order to satisfy the claim of Proposition 1.5.

THEOREM 1.6. Given any element $\alpha$ in $S_{n}, n>2$, (resp. $\left.A_{n}, n>3\right)$, there is an element in $S_{n}\left(A_{n}\right)$ which together with $\alpha$ generates $S_{n}\left(A_{n}\right)$.

Proof $\left({ }^{5}\right)$. Let $\alpha$ be an element of $S_{n}$ that is not the identity. Without loss of generality we may assume that the cycle decomposition of $\alpha$ is

$$
\left(l_{0},+1, \cdots l_{1}\right)\left(l_{1}+1, \cdots, l_{2}\right) \cdots\left(l_{r-1}+1, \cdots, l_{r}\right)
$$

where $l_{0}=0$. Let us first deal with the case $S_{n}$ and $n$ odd. Then we see for $n=3$ that $(1,2)$ and $(1,2,3)$ generate $S_{3}$. Therefore we may assume $n \geqq 5$. In this case let $w_{1}$ and $w_{2}$ be disjoint cycles of length $(n+1) / 2$ resp. $(n-1) / 2$ and choose $w_{1}$ in such a way that it contains the digits $l_{0}+1, \cdots, l_{r-1}+1$, but not all digits of $\alpha$. Then it is obvious that $\alpha$ and $\beta=w_{1} w_{2}$ generate a transitive permutation group $\langle\alpha, \beta\rangle .(n+1) / 2$ and $(n-1) / 2$ are integers that are relatively prime. Therefore we can find integers $a$ and $b$ such that $1+a(n+1) / 2)=b((n-1) / 2)$. Denoting this number by $k$ we see that $\beta^{k}=w_{1}^{k} w_{2}^{k}=w_{1}$, wherefore $w_{1}$ belongs to $\langle\alpha, \beta\rangle$. In order to show that $\langle\alpha, \beta\rangle$ is a primitive group, assume that $M$ is a domain of imprimitivity. Then $M$ cannot contain a digit belonging to $w_{1}$ and at the same time a digit not belonging to $w_{2}$, because in this case all digits of $w_{1}$ would have to be in $M$. Therefore the cardinality of $M$ is a divisor of both $(n+1) / 2$ and $(n-1) / 2$, and hence equals 1 . The primitive group $\langle\alpha, \beta\rangle$ contains a cyclic subgroup, generated by $w_{1}$, that keeps $(n-1) / 2$ digits fixed. Hence a well known result on finite permutation groups [2, p. 207, Theorem I] states that $\langle\alpha, \beta\rangle$ contains the alternating group $A_{n}$ (note that $(n+1) / 2<\frac{2}{3} n$ for $n \geqq 5$ ). But $\beta$ is not an element of $A_{n}$ and thus $\langle\alpha, \beta\rangle=S_{n}$.

If $n \equiv 0$ (2) and if we choose corresponding cycles $w_{1}$ and $w_{2}$ of lengths $n / 2+1$ and $n / 2-1$ (in case $n \equiv 0(4)$ ) resp. $n / 2+2$ and $n / 2-2$ (in case $n \equiv 2$ (4)), we end up with the corresponding result for $A_{n}$ (the cases which have to be checked separately are $n=4,=6$, and $=10$ ). In order to finish the case of the alternating group for $n$ being odd, we choose in the same way as before cycles $w_{1}$ and $w_{2}$ of lengths $(n+1) / 2$ and $(n-3) / 2$ (in case $(n+1) / 2 \equiv 1(2))$ resp. $(n+3) / 2$ and $(n-5) / 2$ (in case $(n+1) / 2 \equiv 0(2))$; here we have only one exceptional case, namely $n=7$.

(5) The idea of this proof is essentially due to H. Zassenhaus. 
What remains to be treated is the case of $S_{n}$ for $n$ being even. Still the old method works in case $\alpha$ is not an element of $A_{n}$. Otherwise we proceed like that: corresponding to whether $n / 2+1 \equiv 1$ (2) or $n / 2+1 \equiv 0$ (2) we construct $w_{1}$ as a cycle of length $n / 2+1$ resp. $n / 2+2$ containing only the digits $1, l_{1}+1, \cdots, l_{r-1}+1$ in this order, and digits $\geqq q+1$, choose $w_{2}$ as a cycle of length $n / 2-3$ resp. $n / 2-6$ which contains at least one (but not all) of the remaining digits $\leqq q$ and is disjoint from $w_{1}$, and put the remaining two resp. four digits into a cycle $w_{3}$. That construction is possible because $\alpha \in A_{n}$ implies $q-r \geqq 2$. Then $\beta=w_{1} w_{2} w_{3}$ has the property that $\langle\alpha, \beta\rangle$ is a transitive permutation group. Again we find a positive integer $k$ so that $k$ is $\equiv 0(2)$ resp. $\equiv 0(4)$ and fulfills

$$
1+a\left(\frac{n}{2}+1\right)=b\left(\frac{n}{2}-3\right)=k \text { resp. } 1+a\left(\frac{n}{2}+2\right)=b\left(\frac{n}{2}-6\right)=k
$$

with suitably chosen integers $a$ and $b$. Therefore we get $\beta^{k}=w_{1}^{k} w_{2}^{k} w_{3}^{k}$ and we can continue as before. This finishes the proof of the theorem except in the exceptional cases mentioned above, in which the statement of the theorem can be verified directly.

Proposition 1.7. Given a set $E$ that is finite or countably infinite. Then there is a transitive subgroup $H$ of $S_{E}$ generated by two elements such that the centralizer of $H$ consists of the identity only.

Proof. For finite sets $E$ the statement is an easy consequence of Theorem 1.6. In case $E$ is countably infinite, let $E$ be the set of integers. Denote by $\alpha$ the cycle $(\cdots,-1,0,1, \cdots)$. Suppose the permutation $\sigma$ commutes with $\alpha$. Then $\sigma$ has to be invariant under transformation by $\alpha$ which implies that $\sigma$ cannot contain a cycle of finite length. If $\sigma$ contains the infinite cycle $(\cdots, a, b, \cdots)$ then this cycle does not change as we add $b-a$ to every entry. Hence it is of the form $(\cdots, a-m, a, a+m, a+2 m, \cdots)$ which implies that $\sigma$ is a suitable power of $\alpha$. However, such a power of $\alpha$ that is not the identity does not commute with any $\beta$ that leaves only the positive integers fixed and permutes the negative integers arbitrarily.

Given a pair $(\alpha, \beta)$ of elements of $S_{E}$ we call every pair of the form $\left(\sigma \alpha \sigma^{-1}, \sigma \beta \sigma^{-1}\right)$ conjugate to the first one. Furthermore, the pair $(\alpha, \beta)$ will be called ample if the subgroup $\langle\alpha, \beta\rangle$ generated by $\alpha$ and $\beta$ fulfills the statement of Proposition 1.7. Then we get the

Corollary. Given a set $E$ that is finite or countably infinite and has cardinality $\geqq 3$. Then there is a set of mutually not conjugate ample pairs of elements in $S_{E}$ whose cardinality is greater or equal to $p(n)$ (if $\operatorname{card} E=n<\aleph_{0}$ ) resp. card $S_{E}$ (if card $E=\aleph_{0}$ ).

Proof. In case $n=3$, we see that the pairs $((1,2),(1,3)),((1,2),(1,2,3))$ and $((1,2,3),(1,2))$ fulfill the requirements. However, for $n \geqq 4$, we choose in every 
conjugacy class of elements of $S_{n}$ that does not contain the identity an element $\alpha$ and construct to it an element $\beta$ with $\langle\alpha, \beta\rangle=S_{n}$ (cf. Theorem 1.6). That gives already $p(n)-1$ mutually not conjugate pairs that are ample. Now take an element $\alpha$ of $A_{n}$ that is not the identity and find $\beta$ such that $\langle\alpha, \beta\rangle=A_{n}$. Then we have a pair which is ample and not conjugate to any of the previous pairs. In case card $E=\aleph_{0}$, we take the statement of the corollary from the proof of Proposition 1.7.

2. Covering spaces of Riemann surfaces. A holomorphic mapping $p: X \rightarrow Y$ of a Riemann surface $X$ onto a connected Riemann surface $Y$ is called an unbounded covering if

(i) $p$ is a locally proper mapping (i.e., to each point $y \in Y$ there is an open neighborhood $U$ so that $p$ restricted to each connected component of $p^{-1}(U)$ is a proper mapping onto $U$ ),

(ii) there is a subset $Y_{0}$ of $Y$ without cluster points in $Y$ so that the induced mapping $p: X-p^{-1}\left(Y_{0}\right) \rightarrow Y-Y_{0}$ is locally topological and every path( $\left.{ }^{6}\right)$ $\phi: I \rightarrow Y-Y_{0}$ can be lifted into each point of $p^{-1}(\phi(0))$.

The set $Y_{0}$ is called a ramification set of $p: X \rightarrow Y$. If there is a ramification set at all, then there is a smallest one; it shall be called the essential ramification set $Y_{e}$ of $p: X \rightarrow Y$. It is obvious that for different points $y \in Y-Y_{e}$ the cardinality of $p^{-1}(y)$ is always the same. In case $X$ is connected, this cardinality is at most $\aleph_{0}$. It is called the degree of the covering $p: X \rightarrow Y$.

Choose a point $y_{*} \in Y-Y_{0}$ and denote the set $p^{-1}(y)$ by $E_{*}$. Each closed path $\phi: I \rightarrow Y-Y_{0}$ that is based in $y_{*}$ (i.e., $\phi(0)=\phi(1)=y_{*}$ ) defines a permutation $\mu_{*}(\phi)$ of $E_{*}$ in the following way: for each element $x$ in $E_{*}$ there is exactly one lifting $\phi_{x}$ of $\phi$ into $x$ (i.e., a path $\phi_{x}: I \rightarrow X$ with $\phi_{x}(0)=x$ and $\phi=p \circ \phi_{x}$ ); its end point $\phi_{x}(1)$ is an element of $E_{*}$; we define now $\mu_{*}(\phi)(x)$ by $\phi_{x}(1)$. It is clear that $\mu_{*}(\phi)$ depends only on the homotopy class of $\phi$ and therefore defines a mapping $\mu_{*}$ of the fundamental group $\pi_{1}\left(Y-Y_{0}, y\right)$ into the permutation group $S_{E_{*}}$ of $E_{*}$. It can be checked easily that

$$
\mu_{*}\left(\phi_{1} \phi_{2}\right)=\mu_{*}\left(\phi_{2}\right) \mu_{*}\left(\phi_{1}\right)\left({ }^{7}\right)
$$

holds. This means that $\mu_{*}$ is an antihomomorphism. We have that $\mu_{*}$ is uniquely determined by the covering $p: X \rightarrow Y$ and the ramification set.

Choosing a standard set $E$ with card $E=$ card $E_{*}$ and a bijective mapping $E_{*}$ $\rightarrow E$, gives rise to an antihomomorphism $\mu: \pi_{1}\left(Y-Y_{0}, y_{*}\right) \rightarrow S_{E}$. Because we may use any bijective mapping $E_{*} \rightarrow E, \mu$ is well determined only up to an inner automorphism of $S_{E}$. $\mu$ is usually called the monodromy of $p: X \rightarrow Y$. Obviously, the class of $\mu$ does not depend on the choice of $y_{*} \in Y-Y_{0}$.

Considering all coverings of $Y$ with ramification set $Y_{0}$ that fulfill card $E_{*}$

(6) I shall denote the closed unit interval $0 \leqq t \leqq 1$.

(7) $\phi_{1}$ and $\phi_{2}$ being paths, $\phi_{1} \phi_{2}$ denotes the path $t \rightarrow \phi_{1}(2 t), 0 \leqq t \leqq 1 / 2$, and $t \rightarrow \phi_{2}(2 t-1)$ $1 / 2 \leqq t \leqq 1$. 
$=$ card $E$ we find that a class (modulo inner automorphisms) of antihomomorphisms $\mu: \pi_{1}\left(Y-Y_{0}, y\right) \rightarrow S_{E}$ determines an unbounded covering $p: X \rightarrow Y$ up to fiber preserving isomorphisms (i.e., bijective mappings $f: X \rightarrow X^{\prime}$ of the total spaces of two coverings $p: X \rightarrow Y, p^{\prime}: X^{\prime} \rightarrow Y$ with $\left.p=p^{\prime} \circ f\right)$ : this has been proved in $[7 ; 12]$ for the case $Y_{0}=\varnothing$ (the finiteness condition in the proof are unnecessary) whence we have a fiber preserving isomorphism over $Y-Y_{0}$; but such an isomorphism is easily seen to be extendable to an isomorphism over $Y$ itself.

Furthermore, we' see that the number of orbits of $\mu\left(\pi_{1}\left(Y-Y_{0}, y_{*}\right)\right)$ on $E$ has the cardinality of the set of connected components of $X$. In particular, $X$ is connected if and only if $\mu\left(\pi_{1}\left(Y-Y_{0}, y_{*}\right)\right)$ acts transitively on $E$.

$Y_{0}$ having no cluster points in $Y$ implies that for each point $y_{0} \in Y_{0}$ there is a simply connected (open) neighborhood $V$ with $V \cap Y_{0}=\left\{y_{0}\right\}$. Evidently, $p^{-1}(V)$ consists of connected components each of which is mapped properly onto $V$ by $p$; thus for each such component $U_{i}$ the cardinality of the corresponding set $E_{i}=p^{-1}\left(y_{0 *}\right) \cap U_{i}, y_{0 *} \in V-\left\{y_{0}\right\}$, is finite. Choosing a path $\phi_{y_{0}}$ from $y_{*}$ to $y_{0 *}$ and a path $\psi_{y_{0}}$ in $V-y_{0}$ based in $y_{0 *}$ whose homotopy class generates $\pi_{1}\left(V-\left\{y_{0}\right\}, y_{0 *}\right)$, we find that every orbit of the cyclic group generated by $\mu\left(\phi_{y_{0}} \psi_{y_{0}} \phi_{y_{0}}^{-1}\right)$ is finite; those orbits correspond in a bijective fashion to the connected components $U_{i}$ and the cardinality of such an orbit equals the cardinality of the corresponding set $E_{i}$. Therefore $y_{0}$ together with $\phi_{y_{0}}$ defines a partition $\pi_{y_{0}, \phi_{y_{0}}}$ of $E$ into finite subsets. For two such partitions corresponding to paths $\phi_{y_{0}}$ resp. $\phi_{y_{0}}^{\prime}$ there is a permutation of $E$ that carries the first partition into the second one. Hence $y_{0}$ determines a class (up to permutations) $\pi_{y_{0}}$ of partitions. $\pi_{y_{0}}$ set is called the local ramification type.

Suppose there is given a connected Riemann surface $Y$, a subset $Y_{0}$ of $Y$ that has no cluster points in $Y$, and a set $E$ with card $E \leqq \aleph_{0}$. We attach to each point $y_{0} \in Y_{0}$ a local ramification type $\pi_{y_{0}}$ of cardinality card $E$ and ask for the cardinality of the set of fiber-isomorphy classes of unbounded and connected coverings $p: X \rightarrow Y$ with ramification set $Y_{0}$ whose local ramification type is the prescribed one for every point $y_{0} \in Y_{0}$. Without loss of generality we may assume that none of the local ramification types $\pi_{y_{0}}$ is trivial, i.e., none of the partitions of $\pi_{y_{0}}$ is the trivial partition. Summing up the previous statements we get

PROPOSITION 2.1 (cf. [6]). The set of fiber-isomorphy classes of unbounded and connected coverings $p: X \rightarrow Y$ with prescribed essential ramification set $Y_{0}$ and prescribed local ramification types $\pi_{y_{0}}, y_{0} \in Y_{0}$, is in a bijective relation with the set of classes of antihomomorphisms $\mu: \pi_{1}\left(Y-Y_{0}, y_{*}\right) \rightarrow S_{E}$ fulfilling

(1) for each point $y_{0} \in Y_{0}$ and $\phi_{y_{0}}, \psi_{y_{0}}$ chosen as above, the local ramification type $\pi_{y_{0}, \phi_{y_{0}}}$ equals the given one, $\pi_{y_{0}}$,

(2) $\mu\left(\pi_{1}\left(Y-Y_{0}, y_{*}\right)\right)$ acts transitively on $E$.

In order to determine the cardinality of the set of classes of antihomomorphisms fulfilling (1) and (2) of Proposition 2.1 we write down a suitable system of gen- 
erators of $\pi_{1}\left(Y-Y_{0}, y_{*}\right)$. In case $Y$ is compact and of genus $g$, we choose a system of retrosections $a_{1}, b_{1}, \cdots, a_{g}, b_{g}$ based in $y$ and denote their homotopy classes by $\alpha_{1}, \beta_{1}, \cdots, \alpha_{g}, \beta_{g} ; Y$ being compact implies that $Y_{0}$ is finite, say $Y_{0}=\left\{y_{1}, \cdots, y_{r}\right\}$. Denoting the homotopy classes of $\phi_{y \rho} \psi_{y_{\rho}} \phi_{y_{\rho}}^{-1}$ by $\gamma_{\rho}$ it is clear, that $\alpha_{1}, \beta_{1}, \cdots, \alpha_{g}, \beta_{g}, \gamma_{1}, \cdots, \gamma_{r}$ form a system of generators of $\pi_{1}\left(Y-Y_{0}, y_{*}\right)$ subject only to the relation

$$
\prod\left\{\alpha_{\sigma} \beta_{\sigma} \alpha_{\sigma}^{-1} \beta_{\sigma}^{-1} \mid \sigma=1, \cdots, g\right\} \cdot \prod\left\{\gamma_{\rho} \mid \rho=1, \cdots, r\right\}=\varepsilon
$$

where $\varepsilon$ denotes the neutral element in $\pi_{1}\left(Y-Y_{0}, y_{*}\right)$.

In case $Y$ is not compact, the fundamental group $\pi_{1}\left(Y-Y_{0}, y_{*}\right)$ is a free group (cf. [1]) and there is a free system of generators of $\pi_{1}\left(Y-Y_{0}, y_{*}\right)$ consisting of the homotopy classes of $\phi_{y_{0}} \psi_{y_{0}} \phi_{y_{0}}^{-1}, y_{0} \in Y_{0}$, and a free system of generators of $\pi_{1}\left(Y, y_{*}\right)$.

If $Y$ is not compact and if the rank of $\pi_{1}\left(Y, y_{*}\right)$ is greater or equal to two, then the above free system of generators contains at least two elements that are not homotopy classes of closed curves $\phi_{y_{0}} \psi_{y_{0}} \phi_{y_{0}}^{-1}, y_{0} \in Y_{0}$. In order to have an antihomomorphism $\mu$ that fulfills the requirements of Proposition 2.1 we prescribe on those two elements as values the components of an ample pair choose as value on the homotopy classes of $\phi_{y_{0}} \psi_{y_{0}} \phi_{y_{0}}^{-1}$ any permutation that gives rise to the prescribed local ramification, and assign to the remaining elements in our system of generators arbitrary values. For every given ample pair we get a bijective correspondence between antihomomorphisms defined in this way (and not only classes of antihomomorphisms) and certain fiber-isomorphy classes of coverings $p: X \rightarrow Y$. Denoting by $p(\operatorname{card} E)$ the cardinality of $S_{E}$ if card $E=\aleph_{0}$ we take from the corollary of Proposition 1.7 that there are at least as many as

$$
p(\operatorname{card} E) \cdot\left(\operatorname{card} S_{E}\right)^{\operatorname{rank}\left(\pi,\left(Y_{1} y_{*}\right)\right)-2} \cdot \prod\left\{N\left(\pi_{y_{0}}\right) \mid y_{0} \in Y_{0}\right\}
$$

possibilities for different choices of $\mu$ which are mutually inequivalent modulo inner automorphisms and which fulfill the requirements of Proposition 2.1. On the other hand, the cardinality of all possible $\mu$ 's is not greater than

$$
\left(\operatorname{card} S_{E}\right)^{\operatorname{rank}\left(\pi_{1}\left(Y, y_{*}\right)\right)} \cdot \prod\left\{N\left(\pi_{y_{0}}\right) \mid y_{0} \in Y_{0}\right\} \text {. }
$$

It is obvious that the latter estimate is still good in case $Y$ is not compact, but simply resp. doubly connected. Summing up we have

Proposition 2.2. Suppose that $Y$ is not compact, $Y_{0}$ a subset of $Y$ without cluster points in $Y$, and that to each element $y_{0} \in Y_{0}$ there is attached a local ramification type $\pi_{y_{0}}$. Then there are at most

$$
p(\operatorname{card} E) \cdot\left(\operatorname{card} S_{E}\right)^{\operatorname{rank}\left(\pi,\left(Y_{1} y_{*}\right)\right)-1} \cdot \prod\left\{N\left(\pi_{y_{0}}\right) \mid y_{0} \in Y_{0}\right\}
$$

non-fiber-isomorphic unbounded coverings of $Y$ with ramification set $Y_{0}$ that have the prescribed local ramification types. In case $\operatorname{rank}\left(\pi_{1}(Y, y)\right) \geqq 2$, there are at least 


$$
p(\operatorname{card} E) \cdot\left(\operatorname{card} S_{E}\right)^{\operatorname{rank}\left(\pi_{1}\left(Y, y_{*}\right)\right)-2} \cdot \prod\left\{N\left(\pi_{y_{0}}\right) \mid y_{0} \in Y_{0}\right\}
$$

such coverings whose total space is connected.

The case of noncompact Riemann surfaces that are simply or doubly connected remains to be treated. For the sake of convenience we index the elements of $Y_{0}$ as $y_{1}, y_{2}, \cdots$. Then we have

COROllary 1. Hypotheses as in Proposition 2.2. Additional hypotheses $\operatorname{rank}\left(\pi_{1}\left(Y, y_{*}\right)\right)=1$ and card $E=n<\aleph_{0}$. Then there are at least

$$
\prod\left\{N\left(\pi_{y \rho}\right) \mid \rho=2, \cdots\right\}
$$

non-fiber-isomorphic unbounded coverings of $Y$ which have connected total space, ramification set $Y_{0}$, and whose local ramification types are the given ones.

Proof. Denoting the homotopy class of $\phi_{y_{1}} \psi_{y_{1}} \phi_{y_{1}}^{-1}$ by $\gamma_{1}$ we fix $\mu\left(\gamma_{1}\right)$ once and forever, yet in such a way that it represents the prescribed local ramification type, and assign to the generator coming from $\pi_{1}\left(Y, y_{*}\right)$ a permutation that forms an ample pair with $\mu\left(\gamma_{1}\right)$. Then we see that regardless what values of $\mu$ we prescribe on the remaining generators we get an antihomomorphism fulfilling the requirements of Proposition 2.1. Obviously, different choices of the values of $\mu$, subject to the restrictions mentioned, give rise to non-fiber-isomorphic coverings. Hence we get the estimate stated in the corollary.

COROllary 2. Hypotheses as in Proposition 2.2. Additional hypotheses $\operatorname{rank}\left(\pi_{1}\left(Y, y_{*}\right)\right)=0$ and card $E=n<\aleph_{0}$. Let $k_{\rho}, \rho=1,2, \cdots$, be the biggest integer $k$ for which $q_{k}\left(\pi_{y \rho}\right) \neq 0$ and denote $q_{k \rho}\left(\pi_{y \rho}\right)$ by $p_{\rho}$. If

$$
\sum\left\{k_{\rho}-1 \mid \rho=1, \cdots, r_{0}\right\} \geqq n-1\left(^{8}\right)
$$

then there are at least

$$
\frac{1}{n !} \prod\left\{\frac{\left(n-k_{\rho}\right) ! k_{\rho} p_{\rho} N\left(\pi_{y \rho}\right)}{n !} \mid \rho=1, \cdots, r_{0}\right\} \cdot \prod\left\{N\left(\pi_{y \rho}\right) \mid \rho=r_{0}+1, \cdots\right\}
$$

non-fiber-isomorphic unbounded coverings of $Y$ which have connected total space, ramification set $Y_{0}$, and whose local ramification types are the given ones.

Proof. Setting $k_{0}=0$ we choose for $\mu\left(\gamma_{\rho}\right), \rho=1, \cdots, r_{0}$ such a product of pairwise disjoint cycles one of which is $\left(k_{0}+\cdots+k_{\rho+1}-\rho+2, \cdots, k_{0}+\cdots+k_{\rho}-\rho+1\right)$ that $\mu\left(\gamma_{\rho}\right)$ presents the prescribed local ramification. These cycles generate already by themselves a transitive permutation group. Therefore, we may choose any

(8) This condition is certainly fulfilled if $Y_{0}$ contains at least $n-1$ elements. 
values of $\mu$ on the remaining generators in order to get an antihomomorphism fulfilling the requirements of Proposition 2.1. Because there are not more than $n$ ! antihomomorphisms in any conjugacy class, we have the estimate of the corollary.

Easy examples show that the condition (10) cannot be sharpened. In case card $E=\aleph_{0}$ and $\operatorname{rank}\left(\pi_{1}\left(Y, y_{*}\right)\right)=1$ one always gets coverings whose total space is connected and whose local ramification types are the given ones. If card $E=\aleph_{0}$ and if $\operatorname{rank}\left(\pi_{1}\left(Y, y_{*}\right)\right)=0$ then there are again such coverings provided the essential ramification set $Y_{0}$ is infinite; however, if the ramification set is finite, then there are examples of local ramification types that cannot be realized by a covering with connected total space.

Now we shall deal with compact Riemann surfaces Y. From (9) we take thate

$$
\prod\left\{\mu\left(\gamma_{\rho}\right) \mid \rho=1, \cdots, r\right\}=\prod\left\{\mu\left(\alpha_{\sigma}^{-1}\right) \mu\left(\beta_{\sigma}\right)^{-1} \mu\left(\alpha_{\sigma}\right) \mu\left(\beta_{\sigma}\right) \mid \sigma=1, \cdots, g\right\} .
$$

The right-hand side of $\left(9^{\prime}\right)$ is an element of the commutator subgroup of $S_{E}$. Hence a necessary condition for the existence of a covering with the required properties will be the existence of permutations $\mu\left(\gamma_{\rho}\right), \rho=1, \cdots, r$, which give the assigned partitions $\pi_{y_{\rho}}$ and fulfill

$$
\prod\left\{\mu\left(\gamma_{\rho}\right) \mid \rho=1, \cdots, r\right\} \in\left[S_{E}, S_{E}\right] .
$$

On account of Proposition 1.2 this condition is empty in case card $E=\aleph_{0}$. In case card $E<\aleph_{0}$, (11) is equivalent to

$$
\Sigma\left\{\Sigma\left\{(k-1) q_{k}\left(\pi_{y_{\rho}}\right) \mid k=1,2, \cdots\right\} \mid \rho=1, \cdots, r\right\}=0 \bmod 2 .
$$

As it turns out, $\left(11^{\prime}\right)$ is "in general" also sufficient for the existence of coverings with connected total space and the prescribed local ramifications.

Proposition 2.3. Let $Y$ be a compact Riemann surface of genus $g, Y_{0}=\left\{y_{1}, \cdots, y_{r}\right\}$ a finite subset of $Y$, and $\pi_{\rho}$ a local ramification type in $y_{\rho}, \rho=1, \cdots, r$. In case card $E=n<\aleph_{0},\left(11^{\prime}\right)$ is a necessary condition for the existence of an unbounded covering of $Y$ with $Y_{0}$ as ramification set that has the prescribed ilocal ramification types. There are at most

$$
\begin{array}{ll}
p(n)^{2}(n !)^{2 g-2} & \text { if } r=0 \text { and } g \geqq 1, \\
p(n)(n !)^{2 g-1} \cdot \prod\left\{N\left(\pi_{\rho}\right) \mid \rho=1, \cdots, r-1\right\} & \text { if } r \geqq 1 \text { and } g \geqq 1, \\
\prod\left\{N\left(\pi_{\rho}\right) \mid \rho=1, \cdots, r-1\right\} & \text { if } r \geqq 1 \text { and } g=0,
\end{array}
$$

non-fiber-isomorphic coverings of $Y$ with the desired properties. If in additio $g \geqq 2$, then there are at least

$$
\begin{array}{ll}
(p(n)-1) q(n)(n !)^{2 g-4} & \text { if } r=0, \\
q(n)(n !)^{2 g-3} \cdot \prod\left\{N\left(\pi_{\rho}\right) \mid \rho=1, \cdots, r-1\right\} & \text { if } r \geqq 1,
\end{array}
$$


non-fiber-isomorphic coverings of $Y$ with the required properties whose total space is connected. In case card $E=\aleph_{0}$ and $g \geqq 2$, there are $\aleph_{0}$ non-fiberisomorphic covering spaces of $Y$ with the required properties whose total space is connected.

Proof. We get an upper bound by assigning to each $\gamma_{\rho}, \rho=1, \cdots, r-1$, a permutation $\mu\left(\gamma_{\rho}\right)$ which defines the desired partition $\pi_{\rho}$, and an arbitrary permutation to each element of the set $\alpha_{1}, \beta_{1}, \cdots, \alpha_{g-1}, \beta_{g-1}$. The values on the remaining two generators, $\alpha_{g}$ and $\beta_{g}$, have to be adjusted so that $\left(9^{\prime}\right)$ is fulfilled. According to Proposition 1.3 we have at most $n ! p(n)$ possibilities for the values of $\mu\left(\alpha_{g}\right)$ and $\mu\left(\beta_{g}\right)$. After prescribing $\mu\left(\gamma_{r}\right)$ within its class we get an upper bound for the number of non-fiber-isomorphic coverings by counting the different possibilities to choose the values of $\mu$ on the set of generators in the way described above. In case $r=0$ we may fix the value of $\mu$ on one of the generators coming from $\pi_{1}\left(Y, y_{*}\right)$, say $\alpha_{1}$; that leaves $p(n)$ possibilities for $\mu\left(\alpha_{1}\right)$. The rest works out as in the case $r \geqq 1$. The lower bound can be found this way. Again we choose appropriate values for $\mu\left(\gamma_{\rho}\right), \rho=1, \cdots, r-1$, arbitrary values for $\mu\left(\alpha_{1}\right), \cdots, \mu\left(\alpha_{g-1}\right)$, $\mu\left(\beta_{1}\right), \cdots, \mu\left(\beta_{g-2}\right)$. Furthermore, we fix a permutation $\mu\left(\gamma_{r}\right)$ representing the given local ramification type at $y_{r}$ and determine $\mu\left(\beta_{g-1}\right)$ in such a way that $\mu\left(\gamma_{r}\right)$ and $\mu\left(\beta_{g-1}\right)$ form an ample pair. Then we choose for $\mu\left(\alpha_{g}\right)$ and $\mu\left(\beta_{g}\right)$ any permutations such that $\left(9^{\prime}\right)$ is fulfilled. Obviously, different choices for $\mu$ within the limitations described give rise to non-fiber-isomorphic coverings with connected total space. That proves the validity of the lower bound for $r \geqq 1$, provided $n \geqq 3$. The case $n=2$ can be handled directly.

In a corresponding way one gets the lower bound in case $r=0$.

The case card $E=\aleph_{0}$ can be worked out in a similar fashion and is left to the reader.

Corollary 1. Hypotheses and notations as in Proposition 2.3 and Corollary 2 of Proposition 2.2. Additional hypotheses $g=1$. If $(10)$ is fulfilled, then there are at least

$$
\frac{1}{n !} q(n) \prod\left\{\frac{\left(n-k_{\rho}\right) ! k_{\rho} p_{\rho} N\left(\pi_{\rho}\right)}{n !} \mid \rho=1, \cdots, r_{0}\right\} \prod\left\{N\left(\pi_{\rho}\right) \mid \rho=r_{0}+1, \cdots, r\right\}
$$

non-fiber-isomorphic coverings of $Y$ with $Y_{0}$ as ramification set that have a connected total space and whose local ramification types are the given ones.

Proof. Combination of the proofs of Proposition 2.3 and Corollary 2 of Proposition 2:2.

In case $Y$ is a compact Riemann surface of genus $g \leqq 1$, then card $E=\aleph_{0}$ does not seem to admit sufficiently general and yet smooth results on the existence of a connected covering space with prescribed local ramification. 
Corollary 2. Hypotheses and notations as in Proposition 2.3 and Corollary 2 of Proposition 2.2. Additional hypotheses $g=0$ and card $E=n<\aleph_{0}$. In case $n=4$, assume that each ramification type $\pi_{\rho}, \rho=1, \cdots, r$, fulfills $q_{2}\left(\pi_{\rho}\right)=2$ and that $r \geqq 3$ holds; then there are exactly $3^{r-1}$ non-fiber-isomorphic coverings of $Y$ with $Y_{0}$ as ramification set whose total space is connected and which have the prescribed local ramification $\left({ }^{9}\right)$. In any other case assume that among the prescribed local ramification types there are some, say $\pi_{1}, \cdots, \pi_{r_{0}}$ with

$$
\sum\left\{k_{\rho}-1 \mid \rho=1, \cdots, r_{0}\right\} \geqq n-1
$$

and that among the remaining local ramification types $\pi_{r_{0}+1}, \cdots, \pi_{r}$ there are $n$ equals ones, say $\pi_{r_{0}+1}, \cdots, \pi_{r_{0}+n}\left(1^{0}\right)$. then there are at least

$$
\frac{1}{n !} \prod\left\{\frac{\left(n-k_{\rho}\right) ! k_{\rho} p_{\rho} N\left(\pi_{\rho}\right)}{n !} \mid \rho=1, \cdots, r_{0}\right\} \prod\left\{N\left(\pi_{\rho}\right) \mid \rho=r_{0}+n+1, \cdots, r\right\}
$$

non-fiber-isomorphic covering spaces of $Y$ with $Y_{0}$ as ramification set whose total space is connected and which have the prescribed local ramification types.

Proof. The proof of the general case of this corollary makes use of Proposition 1.4 and ideas that have been carried out before. The special case $\left(n=4, q_{2}\left(\pi_{\rho}\right)=2\right)$ can be discussed directly. Details are left to the reader.

It is obvious that in any given special situation the results of the first section give much sharper estimates than the ones stated in the propositions and corollaries of this section. For instance, let us discuss the case where $Y$ is a compact Riemann surface of genus 0 , card $E=3$, and for each local ramification type $\pi_{\rho}, \rho=1, \cdots, r$, the relation $q_{3}\left(\pi_{\rho}\right)=1$ holds. The only permutations $\pi$ in $S_{3}$ for which $q_{3}(\pi)=1$ holds are $(1,2,3)$ and $(1,3,2)$. Hence an antihomomorphism is characterized by assigning +1 or -1 to each one of the $r$ points of $Y_{0}$. If we assign +1 to $p$ points and -1 to $q$ points (where $p+q=r$ ), then equation $\left(11^{\prime}\right)$ is always fulfilled and we have

$$
p \equiv 2 r \bmod 3 .
$$

Hence there is always a covering of $Y$ with connected total space and the prescribed local ramification. As the position of the $p$ elements +1 does not affect (12), the number of non-fiber-isomorphic coverings of $Y$ with connected total space and the prescribed local ramification types equals

$$
\Sigma\left\{\left(\begin{array}{c}
r \\
\langle r\rangle+3 l
\end{array}\right) \mid l=0,1, \cdots\right\}
$$

where $\langle r\rangle=2 r-3[2 r / 3]$ is the smallest negative rest of $2 r \bmod 3$. The number of these coverings equals roughly $\frac{1}{3} 2^{r}$.

(9) If $r \leqq 2$, then there are no such coverings.

(10) These conditions are fulfilled if $r \geqq(n=1)(p(n)-1)+1$, as easily verified. 
Other examples can be found in [6] and [7] and articles referred to in these papers. In [6] the case where $Y$ is a compact Riemann surface of genus 0 and $q_{k}\left(\pi_{\rho}\right)=0$ for each $\rho$ and each $k \neq 2$ is discussed completely.

3. Field extensions of algebraic function fields of one variable. Let $K$ be a field of algebraic functions of one variable over the complex number field. We want to investigate the number of nonisomorphic (in the sense of algebra) field extensions $L \supseteq K$ with given degree $[L: K]=n<\aleph_{0}$ and prescribed ramification. The latter means that we fix a finite number of places $Q_{1}, \cdots, Q_{r}$ in $K$ and require that

(i) $Q_{1}, \cdots, Q_{r}$ are the only places which split in the extension $L \supseteq K$,

(ii) $Q_{\rho}, \rho=1, \cdots, r$, splits into $\left.\sum\left\{\sum k P_{\lambda \rho}^{(k)} \mid \lambda=1, \cdots, q_{k \rho}\right\} \mid k=1, \cdots\right\}$ where $q_{k \rho} \geqq 0$ are given integers fulfilling $\sum\left\{k q_{k \rho} \mid k=1, \cdots\right\}=n$.

Without loss of generality we may assume that for every $\rho$ the inequality $\sum\left\{k q_{k \rho} \mid k=2, \cdots\right\} \neq 0$ holds.

$K$ can be regarded as the field of meromorphic functions on some compact Riemann surface $Y$ (which is supposed to be fixed). If we take any compact connected Riemann surface $X$ whose field of meromorphic functions is isomorphic to $L$, then each element of $L$ which generates $L$ over $K$ maps $X$ holomorphically onto $Y$. The resulting unbounded covering $p: X \rightarrow Y$ has degree $n$ and essential ramification set $\left\{y_{1}, \cdots, y_{r}\right\}$ where $y_{\rho}, \rho=1, \cdots, r$, corresponds canonically to $Q_{\rho}$. The ramification type $\pi_{\rho}$ of $p: X \rightarrow Y$ in $y_{\rho}$ is the one given by the set of integers $q_{1 \rho}, q_{2 \rho}, \cdots$. Obviously, nonisomorphic field extensions define non-fiber-isomorphic coverings $p: X \rightarrow Y$ of degree $n$, essential ramification set $\left\{y_{1}, \cdots, y_{r}\right\}$, and local ramification types $\pi_{\rho}$ with $q_{k}\left(\pi_{\rho}\right)=q_{k \rho}$, $k=1,2, \cdots, \rho=1, \cdots, r$. On the other hand, an unbounded covering $p: X \rightarrow Y$ with those properties defines a field extension $L \supseteq K$ which has the required properties. But non-fiber-isomorphic coverings may very well give isomorphic field extensions. Therefore we have to find a criterion which tells us that the function field belonging to non-fiber-isomorphic coverings are not isomorphic.

Suppose $p: X \rightarrow Y$ and $p^{\prime}: X^{\prime} \rightarrow Y$ are unbounded coverings of $Y$ of degree $n$, let the essential ramification set $Y_{e}$ of both coverings be $\left\{y_{1}, \cdots, y_{r}\right\}$, and let the antihomomorphism to which the coverings belong be $\mu$ resp. $\mu^{\prime}$. We say that the ramification points $y_{1}, \cdots, y_{r}$ are in general position if the only biholomorphic mapping of $Y$ onto itself that leaves the set $\left\{y_{1}, \cdots, y_{r}\right\}$ invariant is the identity $\left({ }^{11}\right)$. Suppose there is a biholomorphic mapping $\phi: X \rightarrow X^{\prime}$. It is possible to realize $Y$ as an unbounded covering $q: Y \rightarrow S^{2}$ of the Riemannian sphere which has degree $g+1, g$ being the genus of $Y$. In addition we may assume that $q^{-1}(\infty)$ consists only of one point. Consequently, $f=q \circ p^{\prime} \circ \phi$ is a meromorphic function on $X$ that has order $(g+1) n$. Denoting the branches of $f$ over $Y$ by $f_{1}, \cdots, f_{n}$ the

(11) If $Y$ has genus 0 , then 4 points can be in general position. If the genus of $Y$ is 1 , then 2 points can be in general position. 
polynomial $P(w)=\prod\left(w-f_{v}\right)$ has coefficients in the field $K(Y)$ of meromorphic functions on $Y$; being elementary symmetric functions of the $n$ different branches of $f$, and $\phi$ being a biholomorphic mapping imply that each coefficient is of degree $\leqq(g+1) n$. The poles of these coefficients are contained in the set $p\left(\left(q \circ p^{\prime} \circ \phi\right)^{-1}(\infty)\right)$ which consists of at most $n$ points. The discriminant $\Delta$ of $P(w)$ is therefore a function in $K(Y)$ of degree $\leqq(g+1)(n-1) n^{2}$. Dropping $n$ possible poles for $\Delta$ from the set $Y_{e}$, we are left with at least $r-n$ points of $Y_{e}$ in which $\Delta$ has to be holomorphic. Because $p: X \rightarrow Y$ is ramified in those points, different branches of $f$ have the same value. Consequently, $\Delta$ must vanish in those points. It is classical and easy to calculate from a well-known formula on discriminants that $\Delta$ has in such a point $y_{\rho}$ a zero of order $\geqq \Sigma\left\{(k-1) q_{k}\left(\pi_{\rho}\right) \mid k=1, \cdots\right\}$. If we consider only such coverings for which for every subset $Y_{0 n}$ of $Y_{0}$ containing $r-n$ points

$$
\sum\left\{\Sigma\left\{(k-1) q_{k}\left(\pi_{\rho}\right) \mid k=1, \cdots\right\} \mid Q_{\rho} \in Y_{0 n}\right\} \geqq(g+1)(n-1) n^{2}+1
$$

then we can conclude that $\Delta$ vanishes identically. This means that $P(w)$ is reducible over $K(Y)$. Because $X$ is connected, all irreducible factors of $P(w)$ over $K(Y)$ are the same and we get $P(w)=(Q(w))^{k}$ where $Q(w)$ is a polynomial over $K(Y)$ of degree $d=n / k$ with leading coefficient 1 . Suppose $d>1$. Then we can number the points of $p^{-1}\left(y_{*}\right)=\left\{x_{1}, \cdots, x_{n}\right\}$ in such a way that for every integer $s, 0 \leqq s \leqq k$, the branches of $f$ belonging to the points $x_{s d+1}, \cdots, x_{(s+1) d}$ are the different roots of $Q(w)$. Hence the irreducibility of $Q(w)$ implies that for every element $\alpha \in \pi_{1}\left(Y-Y_{0}, y_{*}\right)$ the permutation $\mu(\alpha)$ maps each set $(s d+1, \cdots,(s+1) d)$, $0 \leqq s \leqq k$, into such a set. This amounts to saying that the monodromy group $\mu\left(\pi_{1}\left(Y-Y_{0}, y_{*}\right)\right)$ is imprimitive. Consequently, if we started out with coverings whose monodromy group is primitive we would have that the degree of $Q(w)$ equals 1. That would mean that for every $y \in Y$ the set $p^{-1}(y)$ is mapped by $f$ into one point of $S^{2}$. Hence $p^{\prime} \circ \phi$ maps $p^{-1}(y)$ into a set whose image under $q$ consists of one point.

We claim that $p^{\prime} \circ \phi\left(p^{-1}(y)\right)$ itself consists of one point for every $y \in Y$. If this is not the case, we would find a point $\bar{y} \in Y$ for which $p^{\prime} \circ \phi\left(p^{-1}(\bar{y})\right)$ contains at least two points, $\bar{y}_{1}$ and $\bar{y}_{2}$. Choose a meromorphic function $q$ on $Y$ that has a pole of order $g+1$ in $\bar{y}_{1}$ and is holomorphic outside of $\bar{y}_{1}$. Then $\bar{q}: Y \rightarrow S^{2}$ is an unbounded covering and the previous argument is still valid. Therefore $\bar{q}\left(p^{\prime} \circ \phi\left(p^{-1}(\bar{y})\right)\right)=\bar{f}\left(p^{-1}(\bar{y})\right)$ must not contain more than one point, which contradicts the hypothesis $\bar{q}\left(\bar{y}_{1}\right) \neq \bar{q}\left(\bar{y}_{2}\right)$.

The fact that for each $y \in Y$ the set $p^{\prime} \circ \phi\left(p^{-1}(y)\right)$ consists of one point makes it possible to define a mapping $\phi_{0}: Y \rightarrow Y$ having the property that $p^{\prime} \circ \phi=\phi_{0} \circ p$. Obviously, $\phi_{0}$ is uniquely determined by this relation. $p^{\prime} \circ \phi$ being holomorphic implies now that $\phi_{0}$ too is a holomorphic mapping (cf. [15]). $\phi_{0}$ is even a biholomorphic mapping. Otherwise there would be two different points $\bar{y}_{1}$ and $\bar{y}_{2}$ 
in $Y$ with $\phi_{0}\left(\bar{y}_{1}\right)=\phi_{0}\left(\bar{y}_{2}\right)$. Without loss of generality we may assume that neither $\bar{y}_{1}$ nor $\bar{y}_{2}$ is a ramification point of $p: X \rightarrow Y$. Hence the bijective mapping $\phi$ would establish an injective mapping of the set $p^{-1}\left(\bar{y}_{1}\right) \cup p^{-1}\left(\bar{y}_{2}\right)$ consisting of $2 n$ points into the set $p^{\prime-1}\left(\phi_{0}\left(\bar{y}_{1}\right)\right)$ containing only $n$ points, which is impossible. We claim furthermore that $\phi_{0}$ maps the set $\left\{y_{1}, \cdots, y_{r}\right\}$ into itself. The points $y_{1}, \cdots, y_{r}$ being ramification points for both, $p: X \rightarrow Y$ and $p^{\prime}: X^{\prime} \rightarrow Y$, are characterized by the property that the cardinality of $p^{-1}\left(y_{\rho}\right)$ (and of $p^{\prime-1}\left(y_{\rho}\right)$ ) is smaller than $n$. Because $\phi$ is a bijective mapping, the cardinality of $\phi\left(p^{-}\left(y_{\rho}\right)\right)$ cannot exceed the cardinality of $p^{-1}\left(\phi_{0}\left(y_{\rho}\right)\right)$. Therefore we have $\phi_{0}^{-1}\left(Y_{0}\right) \subseteq Y_{0}$. Consequently, $\phi_{0}\left(Y_{0}\right)=Y_{0}$ on account of $\phi_{0}$ being bijective. If we assume in addition that the points of $Y_{0}$ are in general position, then $\phi_{0}$ must be the identity mapping.

Now suppose that $g \geqq 2$ and that $\mu\left(\gamma_{r}\right)=\mu^{\prime}\left(\gamma_{r}\right)$ and $\mu\left(\beta_{g-1}\right)=\mu^{\prime}\left(\beta_{g-1}\right)$ form an ample pair. We want to show that under these circumstances $\mu=\mu^{\prime}$. In order to prove this, let $\pi$ be the permutation for which $\phi\left(x_{v}\right)=x_{\pi(v)}^{\prime}$ where $p^{-1}\left(y_{*}\right)=\left\{x_{1}, \cdots, x_{n}\right\}$ and $p^{\prime-1}\left(y_{*}\right)=\left\{x_{1}^{\prime}, \cdots, x_{n}^{\prime}\right\}$. For any element $\delta$ of $\pi_{1}\left(Y-Y_{0}, y_{*}\right)$ and for any integer $v, 1 \leqq v \leqq n$, we can find an element $\eta=\eta(\delta, v)$ in the subgroup generated by $\beta_{g-1}$ and $\gamma_{r}$ such that the permutation $\mu(\delta) \mu(\eta)$ leaves $v$ invariant. Therefore, the path $\eta \delta$ lifted into the point $x_{v}=p^{-1}\left(y_{*}\right)$ is a closed path $\delta$. Hence $\phi(\delta)$ is again a closed path in $X^{\prime}$ which lies above $\eta \delta$ and is based in $x_{\pi(v)}^{\prime}$. Thus both, $\mu(\delta) \mu(\eta)$ and $\pi^{-1} \mu^{\prime}(\delta) \mu^{\prime}(\eta) \pi$ map $v$ into the same integer. That holds for every $v, 1 \leqq \nu \leqq n$, whence $\mu=\pi^{-1} \mu^{\prime} \pi$. Yet, $\mu^{\prime}\left(\beta_{g-1}\right)$ and $\mu^{\prime}\left(\gamma_{r}\right)$ form an ample pair wherefore $\pi$ must be the identity.

In the proof of Proposition 2.3 the lower bound for the number of non-fiberisomorphic covering spaces with connected total space and prescribed local ramification type was gotten (in case $g \geqq 2$ ) by constructing certain covering spaces for which $\mu\left(\gamma_{r}\right)$ was fixed within its conjugacy class and $\mu\left(\beta_{g-1}\right)$ chosen in such a way that it formed an ample pair with $\mu\left(\gamma_{r}\right)$, while the values of $\mu$ on the remaining generators could be chosen arbitrarily, yet subject to the condition that $\left(9^{\prime}\right)$ was fulfilled. The ample pairs we used then were the ones taken from the corollary of Proposition 1.6 which insures that the monodromy group of the covering space is primitive. Therefore, the previous argument shows that all these non-fiber-isomorphic coverings define nonisomorphic field extensions provided the above mentioned requirements concerning the points $Q_{\rho}$ are fulfilled. Therefore we get

THEOREM 3.1. Let $K$ be an algebraic function field of one variable over the complex number field and assume that the genus $g$ of $K$ is bigger than or equal to 2. Let $Q_{1}, \cdots, Q_{r}$ be places in $K$ that are in general position and assign to each $Q_{\rho}, \rho=1, \cdots, r, a$ (nontrivial) ramification type $\pi_{\rho}$. If every subset $Y_{0 n}$ of $\left\{Q_{1}, \cdots, Q_{r}\right\}$ that has $r-n$ elements fulfills the condition $\left({ }^{12}\right)$

(12) This condition is certainly fulfilled provided $r \geqq(g+1)(n-1) n^{2}+n+1$. 


$$
\sum\left\{\Sigma\left\{(k-1) q_{k}\left(\pi_{\rho}\right) \mid k=2, \cdots\right\} \mid Q_{\rho} \in Y_{0 n}\right\} \geqq(g+1)(n-1) n^{2}+1
$$

then there are at least

$$
q(n)(n !)^{2 g-3} \prod\left\{N\left(\pi_{\rho}\right) \mid \rho=1, \cdots, r-1\right\}
$$

nonisomorphic field extensions $L \supseteq K$ of degree $n$ that have exactly the ramifications prescribed by the $\pi_{\rho}$ 's.

The proof of this theorem assumed tacitly $n \geqq 3$. However, a direct calculation shows that this estimate is still valid for $n=2$. In addition it may be remarked that an upper bound for the number of nonisomorphic field extensions with the desired properties can be taken immediately from Proposition 2.3.

The cases $g=0$ and $g=1$ are more difficult to handle. The reason is that we may not have enough primitive monodromy groups among the ones which are available for constructing the required covering spaces. Still there is an easy case, namely $n$ being prime. Then the monodromy group is primitive under any circumstances, whence we get

Corollary 1. Hypotheses as in Theorem 3.1, except $g \leqq 1$. Additional hypothesis: $n$ is a prime number. Then the number of nonisomorphic field extensions with the required properties has as lower bound

(a) if $g=1$ and if $\sum\left\{k_{\rho}-1 \mid \rho=1, \cdots, r_{0}\right\} \geqq n-1$ is fulfilled:

$$
\frac{1}{n !} q(n) \prod\left\{\frac{\left(n-k_{\rho}\right) ! k_{\rho} p_{\rho} N\left(\pi_{\rho}\right)}{n !} \mid \rho=1, \cdots, r_{0}\right\} \prod\left\{N\left(\pi_{\rho}\right) \mid \rho=r_{0}+1, \cdots, r\right\},
$$

(b) if $g=0, \Sigma\left\{k_{\rho}-1 \mid \rho=1, \cdots, r_{0}\right\} \geqq n-1$ and if among the ramification types $\pi_{r_{0}+1}, \cdots, \pi_{r}$ there are at least $n$ equal ones, say $\pi_{r_{0}+1}, \cdots, \pi_{r_{0}+n}$ :

$$
\frac{1}{n !} \prod\left\{\frac{\left(n-k_{\rho}\right) ! k_{\rho} p_{\rho} N\left(\pi_{\rho}\right)}{n !} \mid \rho=1, \cdots, r_{0}\right\} \prod\left\{N\left(\pi_{\rho}\right) \mid \rho=r_{0}+n+1, \cdots, r\right\} .
$$

Again, upper bounds can be taken from the corollaries of Proposition 2.3.

Proof. In either case we assume that the values of $\mu\left(\gamma_{1}\right), \cdots, \mu\left(\gamma_{r_{0}}\right)$ are for both coverings, $p: X \rightarrow Y$ and $p^{\prime}: X^{\prime} \rightarrow Y$, chosen as in the proof of Corollary 2 of Proposition 2.2. Then we see as in the proof of Theorem 3.1 that isomorphy of the function fields implies $\mu=\pi^{-1} \mu^{\prime} \pi$, wherefore the method of obtaining lower bounds in Corollaries 1 and 2 of Proposition 2.3 leads to the lower bounds given in this corollary.

A consequence of the proof of Corollary 1 of Theorem 3.1 is that in the example discussed at the end of $\$ 2$ non-fiber-isomorphic coverings always define nonisomorphic field extensions. The same is true for the case discussed in (7) provided (notation as in (7)) $S=(1,2, \cdots, n)$.

The remaining cases can be worked out by means of Proposition 1.5. Here we have 
Corollary 2. Hypotheses as in Theorem 3.1, except $g \leqq 1$. Additional hypothesis: $n$ is neither 4 nor a prime. Then the number of nonisomorphic field extensions with the required properties has as lower bound

(a) if $g=1, \sum\left\{k_{\rho}-1 \mid \rho=1, \cdots, r_{0}\right\} \geqq n-1$, and if among the remaining ramification types $\pi_{r_{0}+1}, \cdots, \pi_{r}$ there are at least $[(n+3) / 2]$ equal ones $\left({ }^{13}\right)$, say $\pi_{r_{0}+1}, \cdots, \pi_{r_{0}+[(n+3) / 2]}$ :

$\frac{q(n)}{n\left[\frac{n+1}{2}\right] !} \prod\left\{\frac{\left(n-k_{\rho}\right) ! k_{\rho} p_{\rho}}{n !} N\left(\pi_{\rho}\right) \mid \rho=1, \cdots, r_{0}\right\} \cdot \prod\left\{N\left(\pi_{\rho}\right) \mid \rho=r_{0}+\left[\frac{n+3}{2}\right]+1, \cdots, r\right\}$,

(b) if $g=0, \sum\left\{k_{\rho}-1 \mid \rho=1, \cdots, r_{0}\right\} \geqq n-1$, and if the set of remaining ramification types $\pi_{r_{0}+1}, \cdots, \pi_{r}$ contains two disjoint subsets of cardinality $n$ resp. $[(n+3) / 2]$ each of which consists of equal ramification types $\left({ }^{14}\right)$ say $\left\{\pi_{r_{0}+1}, \cdots, \pi_{r_{0}+[(n+3) / 2]}\right\}$ and $\left\{\pi_{r_{0}+[(n+3) / 2]+1}, \cdots, \pi_{r_{0}+[(n+3) / 2]+n}\right\}:$

$$
\begin{aligned}
\frac{1}{n\left[\frac{n+1}{2}\right] !} \prod\left\{\frac{\left(n-k_{\rho}\right) ! k_{\rho} p_{\rho}}{n !} N\left(\pi_{\rho}\right) \mid \rho=1, \cdots, r_{0}\right\} \\
\cdot \prod\left\{N\left(\pi_{\rho}\right) \mid \rho=r_{0}+\left[\frac{n+3}{2}\right]+n+1, \cdots, r\right\} .
\end{aligned}
$$

Proof. Again $\mu\left(\gamma_{1}\right), \cdots, \mu\left(\gamma_{r_{0}}\right)$ are chosen as in the proof of Corollary 2 of Proposition 2.2. That makes the monodromy group of the covering a transitive group. The set of ramification types that contains $[(n+3) / 2]$ equal elements is used for getting an element in the monodromy group that makes the monodromy group primitive (cf. Proposition 1.5). The length of the cycles constructed in Proposition 1.5 is always $\geqq(n-1) / 2$. Therefore we have at least $1 / n \cdot n ! /[(n+1) / 2]$ ! different possibilities of choosing $\mu\left(\gamma_{r_{0}+1}\right), \cdots, \mu\left(\gamma_{r_{0}+[(n+3) / 2]}\right)$ so that their product has the property claimed in Proposition 1.5. Now we may proceed as before in order to obtain the estimates stated in the corollary.

In case $n=4$ we again assume the hypotheses of Theorem 3.1, except $g \leqq 1$. Provided at least one ramification type is not conjugate to $(1,2)(3,4)$, then the estimates of our last corollary still hold (they could even be improved slightly). However, if all ramification types are conjugate to $(1,2)(3,4)$, we take from the proof of Theorem 3.1 that the polynomial $Q(w)$ which is annihilated by $f$ has degree 2 and leading coefficient 1 ; its coefficients being elements of $K(Y)$ are of degree 2 resp. 4 and its discriminant is of order 4 . If the monodromy is now chosen in such a way that two different elements of Klein's Vierergruppe turn up in every set of ramification types belonging to $Y_{04}$ at least five times, then the

(13) These hypotheses are fulfilled if $r \geqq[(n+3) / 2](p(n)-1)+1$.

(14) These hyptheses are fulfilled provided $r \geqq(n-1)(p(n)-1)+1$ holds. 
discriminant of the above polynomial must vanish identically (this condition can certainly be fulfilled if $r \geqq 20$ ). This in turn implies that $f$ annihilates a linear polynomial over $K(Y)$, and we may continue as in the previous cases. By means of this observation one can again find lower bounds for the number of nonisomorphic field extensions; for instance $3^{r-20} \cdot 18 ! /(6 !)^{3}$ is such a lower bound.

It is quite obvious that the corresponding questions concerning noncompact Riemann surfaces and their fields of meromorphic functions is much harder to deal with.

4. Ring extensions induced by covering mappings. Let $p: X \rightarrow Y$ be a holomorphic mapping of a connected Riemann surface $X$ into a connected Riemann surface $Y$. Denote the integral domain of holomorphic (field of meromorphic) functions on $X$ by $I(X)(K(X))$. Then $p$ induces a natural homomorphism $p^{*}: I(Y) \rightarrow I(X)(K(Y) \rightarrow K(X))$. In case $p(X)$ consists of more than one point $p^{*}$ is an injective mapping. This makes $I(Y)(K(Y))$ a subring (subfield) of $I(X)$ $(K(X))$. The canonical question which arises here is to determine the structure of the extension $I(X) \supseteq I(Y)$ resp. $K(X) \supseteq K(Y)$. We restrict ourselves to the case where $p: X \rightarrow Y$ is an unbounded covering of finite degree.

In case $Y$ is compact a classical result states:

Proposition 4.1. If the degree of the unbounded covering $p: X \rightarrow Y$ equals $n$, then the degree of the algebraic field extension $K(X) \supseteq K(Y)$ equals $n$. To each algebraic field extension $F$ of $K(Y)$ of finite degree there is an unbounded covering $p: X \rightarrow Y$ so that the field extension $K(X) \supseteq K(Y)$ is isomorphic to the extension $F \supseteq K(Y)$ (i.e., there is an isomorphism which maps $K(Y)$ identically). Given two unbounded coverings of finite degree, $p: X \rightarrow Y$ and $p^{\prime}: X^{\prime} \rightarrow Y$, a fiber mapping $f: X \rightarrow X^{\prime}$ (i.e., a holomorphic mapping with $p=p^{\prime} \circ f$ ) induces a homomorphism $f^{*}: K\left(X^{\prime}\right) \rightarrow K(X)$ that preserves $K(Y)$; to each injective homomorphism $\phi: K\left(X^{\prime}\right) \rightarrow K(X)$ that preserves $K(Y)$ there is exactly one fiber mapping $f: X \rightarrow X^{\prime}$ with $\phi=f^{*}$.

We raise the corresponding questions concerning $I(Y)$ for noncompact Riemann surfaces $Y$. Here we have

THEOREM 4.2. Suppose $Y$ is a noncompact Riemann surface and $p: X \rightarrow Y$ an unbounded covering of finite degree $n$. Then $I(X)$ is a ring extension of $I(Y)$ that has no zero divisors, is integrally closed, and is a free $I(Y)$-module of dimension $n$. On the other hand, given a ring extension $A \supseteq I(X)$ where $A$ has no zero divisors, is integrally closed, and is a free $I(Y)$-module of dimension $n$, there is an unbounded covering $p: X \rightarrow Y$ and an isomorphism $j: I(X) \rightarrow A$ so that $j \circ p^{*}$ equals the inclusion of $I(Y)$ in $A . p: X \rightarrow Y$ is uniquely determined up to a fiber isomorphism.

Proof. In $[8 ; 11 ; 12]$ for a compact Riemann surface $Y$ and an unbounded 
covering $p: X \rightarrow Y$ of degree $n$ a holomorphic fiber bundle of rank $n, p_{*}(1) \rightarrow Y$, is constructed so that there is a natural bijective correspondence between the set of holomorphic (meromorphic) sections over an open subset $U$ of $Y$ and the set of holomorphic (meromorphic) functions on $p^{-1}(U)$. The same construction can be carried out in case $Y$ is not compact. The principal bundle associated with $p(1)_{*}$ is holomorphically trivial $[4 ; 11]$. That, together with the construction of $p_{*}(1)$, implies that there are $n$ holomorphic functions on $X$ which form an $I(Y)$-basis of $I(X)$ (cf. [12]). The fact that $I(X)$ has no zero divisors is equivalent to the hypothesis that $X$ is connected. The statement that $I(X)$ is integrally closed follows trivially from the fact that the ring of convergent power series is integrally closed.

Now to the second part of Theorem 4.2. The field of meromorphic functions on $Y$ is known to be the field of quotients of $I(Y)$ (cf. [3]). Let us denote the field of quotients of $A$ by $F$. Clearly we have an injective homomorphism $K(Y) \subseteq F$ that extends the inclusion $I(Y) \subseteq A$. Therefore we may assume that $K(Y) \subseteq F$ in such a way that this inclusion preserves the inclusion $I(Y) \subseteq A$ (as usual a ring without zero divisors is considered as a subring of its field of quotients). Again, $F$ is a free $K(Y)$-module of dimension $n: F$ is certainly a finite field extension of $K(Y)$ and therefore it is an immediate consequence of [9, Satz 74] that an $I(Y)$-basis of $A$ serves also as a $K(Y)$-basis of $F$. Now we choose a generating element of $F$ over $K(Y)$. It annihilates an irreducible polynomial over $K(Y)$ of degree $n$ that has leading coefficient 1 . This polynomial defines in the usual way an unbounded covering $p: X \rightarrow Y$ so that we have an isomorphism $j_{0}: F \rightarrow K(X)$ whose restriction to $K(Y)$ equals $p^{*} . j_{0}(A)$ is a subring of $K(X)$ and, denoting the restriction of $j_{0}$ to $A$ by $j$, we have the commutative diagram

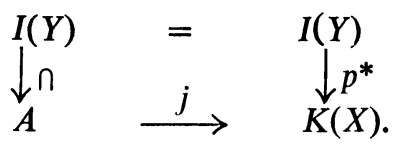

We want to get information on $j(A)$. Firstly we claim that $j(A) \subseteq I(X)$. Otherwise there would be an element $a \in A$ for which $j(a)$ has a pole, say of order $m$ $(m \geqq 1)$. Then $a^{q}, q=1,2, \cdots$, would have an image $j\left(a_{q}\right)$ that has a pole of order $m q$. But this contradicts the hypothesis that $A$ is a finitely generated $I(Y)$-module. The fact that $F$ is the field of quotients of $A$ and that $j_{0}$ is an isomorphism implies that $K(X)$ is the field of quotients of $j(A)$. In order to show that $j(A)=I(X)$ we choose $n$ elements, $a_{1}, \cdots, a_{n}$, in $A$ which constitute an $I(Y)$-basis of $A$. $f_{1}=j\left(a_{1}\right), \cdots, f_{n}=j\left(a_{n}\right)$ being holomorphic functions on $X$ define global sections $s_{1}, \cdots, s_{n}$ in the bundle $p_{*}(1)$. Denoting by $p_{*}^{n}(1)$ the bundle associated with $p_{*}(1)$ which has the space of all complex $n \times n$ matrices as fiber (on which $\operatorname{GL}(n, C)$ operates by right multiplication) the sections $s_{1}, \cdots, s_{n}$ define a nonsingular holomorphic section $\sigma$ in $p_{*}^{n}(1)$. If we can prove that this section $\sigma$ is nowhere singular, 
then $\sigma$ actually is a section in the principal bundle associated with $p_{*}(1)$. Hence $f_{1}, \cdots, f_{n}$ would form an $I(Y)$-basis of $I(X)$ which would imply $j(A)=I(X)$.

From the construction of $p_{*}(1)$ as described in (12) we take that the section in $p_{*}^{n}(1)$ defined by $s_{1}, \cdots, s_{n}$ is nonsingular in $y \in Y$ if and only if the following condition is fulfilled:

Let $p^{-1}(y)=\left\{x_{1}, \cdots, x_{m}\right\}$ and $x_{\mu}, \mu=1, \cdots, m$, be a ramification point of order $l_{\mu}$ (hence $l_{1}+\cdots+l_{m}=n$ ), then the determinant $\Delta(y)$ whose entries are the $n$ branches of $f_{1}, \cdots, f_{n}$ has exact order $\frac{1}{2} \sum\left\{l_{\mu}\left(l_{\mu}-1\right) \mid \mu=1, \cdots, m\right\}=\omega(y)$ in $y$.

Because $\sigma$ is a nonsingular holomorphic section in $p_{*}^{n}(1), \Delta(y)$ has an order $\geqq \omega(y)$ for every point $y \in Y$. In order to show that we actually have equality, let $y$ be any point in $Y$. Then there are holomorphic functions $\tilde{f}_{\lambda \mu}$ on $X, \mu=1, \cdots$, $m, \lambda=0, \cdots, l_{\mu}-1$, which have a power series development $\delta_{\rho \mu} t_{\rho}^{\lambda}+t_{\rho}^{n}+\cdots$ in $x_{\rho} \in p^{-1}(y)$ where $t_{\rho}$ is a local uniformizer in $x_{\rho}$; the existence of such functions is well known [3]. Every one of these functions is integral over $j(A)$ because it annihilates a polynomial of degree $n$ over $I(Y)$ with leading coefficient 1 . Moreover each function $\tilde{f}_{\lambda \mu}$ belongs to $K(X)$ which was the quotient field of $j(A)$. $A$ being integrally closed implies therefore $\tilde{f}_{\lambda \mu} \in j(A)$. For $j(A)$ is generated as an $I(Y)$-module by $f_{1}, \cdots, f_{n}$ there are holomorphic functions $h_{1 \lambda \mu}, \cdots, h_{n \lambda \mu} \in I(Y)$ with

$$
\tilde{f}_{\lambda \mu}=h_{1 \lambda \mu} f_{1}+\cdots+h_{n \lambda \mu} f_{n}, \quad \mu=1, \cdots, m, \lambda=0, \cdots, l_{\mu}-1 .
$$

Therefore, the determinant $D$ having the branches of the $\tilde{f}_{\lambda \mu}$ as entries must have an order in $y$ which is greater or equal than the order of $\omega(y)$. On the other hand, the order of $D$ can easily be calculated: it equals $\omega(y)$. Consequently, the order of $\Delta(y)$ cannot be bigger than $\omega(y)$, and therefore equals $\omega(y)$.

The remaining claim of Theorem 4.2, namely, that $p: X \rightarrow Y$ is uniquely determined up to a fiber isomorphism, follows now immediately. If $p^{\prime}: X^{\prime} \rightarrow Y$ is another covering with the required properties, then a well-known theorem (13) on homomorphisms of rings of holomorphic functions states the existence of a biholomorphic mapping $f: X \rightarrow X^{\prime}$ such that $f^{*}=j^{-1} \circ j^{\prime}$ holds where $j^{\prime}$ denotes the isomorphism $I\left(X^{\prime}\right) \rightarrow A$ described in Theorem 4.2. $f^{*}$ leaves $I(Y)$ invariant. In order to see that $f$ maps every set $p^{-1}(y)$ onto the set $p^{\prime-1}(y)$, take a holomorphic function $h$ in $Y$ that vanishes only in $y$ (cf. [3]). The equation $f^{*}(h)=h$ means $h \circ p^{\prime} \circ f=h \circ p$. Because $h$ vanishes only in $y$, the last equation implies $f\left(p^{-1}(y)\right)=p^{\prime-1}(y)$. Hence $f$ is a fiber mapping.

From Theorem 4.2 and (13) one derives at once in the same way

Corollary 1. Suppose $I(Y)$ is the ring of holomorphic functions on a noncompact Riemann surface $Y$. Let $A$ and $A^{\prime}$ be ring extensions of $I(Y)$ both fulfilling the conditions of Theorem 4.2 and let $\phi: A \rightarrow A^{\prime}$ be a homomorphism which leaves $I(Y)$ invariant. Then there is exactly one holomorphic fiber mapping $f: X^{\prime} \rightarrow X$ of the total spaces of the corresponding unbounded coverings, $p: X \rightarrow Y$ and $p^{\prime}: X^{\prime} \rightarrow Y$, with $\phi=f^{*}$. Consequently, $\phi$ is injective. 
COROLLARY 2. Let $Y$ be a noncompact Riemann surface and $F$ a finite algebraic field extension of the field $K(Y)$ of meromorphic functions on $Y$. Then the integral closure $A$ of $I(Y)$ in $F$ is integrally closed, has $F$ as its field of quotients and is a free $I(Y)$-module of dimension $[F: K(Y)]$.

Proof. $F$ is a simple algebraic extension and therefore there is an unbounded covering $p: X \rightarrow Y$ and an isomorphism $j: F \rightarrow K(X)$ whose restriction to $K(Y)$ equals $p^{*}$. The integral closure $A$ of $I(Y)$ in $K(X)$ is obviously a subring of the ring $I(X)$ and hence coincides with $I(X)$. Because $p: X \rightarrow Y$ is an unbounded covering of degree $[F: K(Y)]$ everything follows immediately from Theorem 4.2.

From the last corollary we get at once

Corollary 3. Let $Y$ be a noncompact Riemann surface and $A$ be a finite algebraic ring extension of $I(Y)$ which is integral over $I(Y)$. Then $A$ is $I(Y)-$ isomorphic to a subring of the ring of holomorphic functions on some unbounded covering of $Y$.

Another question related to the one dealt with in Theorem 4.2 is the following. Let $Y_{0}$ be a (compact or noncompact) connected Riemann surface and $Y$ a connected, open, and proper subset of $Y_{0}$. Then we denote by $I\left(Y, Y_{0}\right)$ the intersection $I(Y) \cap K\left(Y_{0}\right)$. An unbounded covering $p:\left(X, X_{0}\right) \rightarrow\left(Y, Y_{0}\right)$ is meant to be an unbounded covering $p: X_{0} \rightarrow Y_{0}$ for which $x=p^{-1}(Y)$; hence $p \mid X: X \rightarrow Y$ is again an unbounded covering and both, $p: X_{0} \rightarrow Y_{0}$ and $p \mid X: X \rightarrow Y$, have the same degree. Then we get

THEOREM 4.3. Let $p:\left(X, X_{0}\right) \rightarrow\left(Y, Y_{0}\right)$ be an unbounded covering of finite degree $n$ and $Y_{0}$ either noncompact or the Riemannian sphere. Then $I\left(X, X_{0}\right)$ is a ring extension of $I\left(Y, Y_{0}\right)$ that has no zero divisors, is integrally closed, and is a free $I\left(Y, Y_{0}\right)$-module of dimension $n$. On the other hand, given a ring extension $A \supseteq I\left(Y, Y_{0}\right)$ with these properties, there is an unbounded covering $p:\left(X, X_{0}\right) \rightarrow\left(Y, Y_{0}\right)$ of degree $n$ and an isomorphism $j: I\left(X, X_{0}\right) \rightarrow A$ so that $j \circ p^{*}$ equals the inclusion of $I\left(Y, Y_{0}\right)$ in $A . p:\left(X, X_{0}\right) \rightarrow\left(Y, Y_{0}\right)$ is uniquely determined up to a fiber isomorphism.

Proof. The first part of the theorem follows from the proof of the first part of Theorem 4.2 in case $Y_{0}$ is not compact. In case $Y_{0}$ is the Riemannian sphere we pick a point $y_{0} \in Y_{0}-Y$. It will be sufficient to show that $p_{*}^{n}(1)$ admits a meromorphic section which is holomorphic and nowhere singular over $Y_{0}-\left\{y_{0}\right\}$. But that means only the existence of a so-called normal basis of the ideal of meromorphic functions in $Y_{0}$ which are holomorphic in $Y_{0}-\left\{y_{0}\right\}$. The existence of a normal basis is classical, but could also be concluded easily from (11).

As to the second part of the statement. It is clear that $K(Y)$ is the quotient field of $I\left(Y, Y_{0}\right)$ regardless whether $Y_{0}$ is compact or not: in the first case it is a trivial consequence of the Riemann-Roch theorem, in the latter one it follows 
from (3). In either case we proceed now as in the proof of the second part of Theorem 4.2. The only thing we have to be sure of is the existence of the functions $\tilde{f}_{\lambda \mu}$. In case $Y_{0}$ is not compact, we use the functions employed in the proof of Theorem 4.2. In case $Y_{0}$ is compact, the existence of such functions is classical. That amounts to the existence of an unbounded covering $p:\left(X, X_{0}\right) \rightarrow\left(Y, Y_{0}\right)$ and an isomorphism $j: I\left(X, X_{0}\right) \rightarrow A$ with the required properties. What remains to be proved is the uniqueness part. Suppose we have two such coverings, $p:\left(X, X_{0}\right) \rightarrow\left(Y, Y_{0}\right)$ and $p^{\prime}:\left(X^{\prime}, X_{0}^{\prime}\right) \rightarrow\left(Y, Y_{0}\right)$, and isomorphisms $j$ and $j^{\prime}$. $j$ and $j^{\prime}$ define an isomorphism $\psi: I\left(X^{\prime}, X_{0}^{\prime}\right) \rightarrow I\left(X, X_{0}\right)$ which leaves $I\left(Y, Y_{0}\right)$ invariant. This isomorphism has a unique extension to an isomorphism of $K\left(X^{\prime}\right)$ onto $K(X)$ which again shall be denoted by $\psi$. Next we see that the points of $X$ correspond bijectively to the set of all maximal principal ideals in $I\left(X, X_{0}\right)$ : this is classical in case $Y_{0}$ is compact and follows similarly to the analogous statement in (13). Therefore, there is a bijective mapping $f: X \rightarrow X^{\prime}$ so that for every $a \in I\left(X^{\prime}, X_{0}^{\prime}\right)$ and every $x \in X$ the equation

$$
a(x)=\psi(a)(f(x))
$$

is fulfilled. Because $K\left(X^{\prime}\right)$ is the quotient field of $I\left(X^{\prime}, X_{0}^{\prime}\right)$, that equation holds for every function in $K\left(X^{\prime}\right)$. Therefore, the set described in [5], Theorem A, is not empty and, consequently, there is exactly one holomorphic mapping $f_{0}: X_{0} \rightarrow X_{0}^{\prime}$ with $\psi=f_{0}^{*}$. Interchanging $X$ and $X^{\prime}$ we see that $f_{0}$ is a biholomorphic mapping which can be checked easily to be a fiber mapping.

REMARK. The second part of Theorem 4.3 is true for any compact Riemann surface $Y_{0}$ as the proof of this part does not use the hypothesis that $Y_{0}$ is the Riemannian sphere. The first part of Theorem 4.2, however, is valid only for $Y_{0}$ being a noncompact Riemann surface resp. $Y_{0}$ being the Riemannian sphere, as can be shown by counter-examples.

5. Final remarks. 1. In $\S 2$ we discussed field extensions of algebraic function fields of prescribed degree and prescribed local splitting. From the point of view of field extensions it seems to be perhaps more interesting to look for field extensions which have given degree, a given Galois group, and given splitting groups. It is no restriction to assume that the Galois group is given as a permutation group. In this case the degree of the field extension is given implicitly. Prescribing the splitting groups means now that we prescribe not only the ramification types, but moreover the values of the permutations $\mu\left(\gamma_{\rho}\right)$. The geometric situation corresponding to this problem is to construct unbounded coverings whose ramification set $Y_{0}$ as well as the monodromy group (= Galois group of the field extension) and the values of the permutations $\mu\left(\gamma_{\rho}\right)$ are given. In order to get again estimates on the number of non-fiber-isomorphic coverings resp. nonisomorphic field extensions realizing the given situation we proceed basically 
in the same way as in $\S 2$ and $\S 3$, except that we are not free anymore to choose the values $\mu\left(\gamma_{\rho}\right)$ suitably and the values $\mu\left(\alpha_{\lambda}\right)$ resp. $\mu\left(\beta_{\lambda}\right)$ quite arbitrarily (the latter ones have to be elements of the given Galois group). In the general case, that is the case covered by Proposition 3.2, we have to find a substitute for the use of Proposition 1.3. This substitute is to determine for the given Galois group $G$ the smallest number $k(G)$ such that every element of the commutator subgroup $[G, G]$ of $G$ can be written as product of $k(G)$ commutators in $G$. In addition we have to find a lower bound $q(G)$ for the number of possibilities in which every element of $[G, G]$ can be written as product of $k(G)$ commutators in $G$. It may be remarked that $k(G)$ can assume arbitrarily big values. Furthermore, we have to determine the length $l(G)$ of a minimal system of generators of $G$ and a lower bound $p(G)$ for the number of conjugacy classes of systems of generators of length $l(G)$. Again it can be shown that $l(G)$ can assume arbitrarily big values. The methods of $\$ 2$ show now that there are at least

$$
q(G) \cdot(l(G)) ! \frac{|C(G)|}{|N(G)|}|G|^{2 g-2 k(G)-l(G)}
$$

non-fiber-isomorphic coverings with the desired properties, provided the genus $g$ of $Y$ is greater or equal to

$$
k(G)+\left[\frac{l(G)+1}{2}\right]
$$

here $|G|$ is the order of $G, C(G)$ the centralizer of $G$, and $N(G)$ the normalizer of $G$. In case the Galois group $G$ is primitive, $\S 3$ can be carried over to our situation immediately. Hence, under the additional hypotheses of Theorem 3.1 (13) is also a lower bound for the number of nonisomorphic field extensions that have the prescribed ramification set, Galois group, and splitting groups. If $G$ happens to be imprimitive one has to modify the methods of $\$ 3$ suitably in order to still get reasonable estimates. The details are left to the reader.

2. If we want to apply the methods of this paper to the case of higher dimensional complex spaces we run into serious difficulties. Still, one can see easily that there are only finitely many field extensions of the field of meromorphic functions on a compact complex space whose degree and ramification types are prescribed. But that is all one can expect in the general case for the fundamental group of a compact complex space minus a purely one-codimensional analytic subset can be quite complicated. If in a special situation the fundamental group is known explicitly, the methods developed in this paper lead to reasonable lower and upper bounds for the number of nonisomorphic field extensions with the required properties. 


\section{REFERENCES}

1. L. V. Ahlfors and L. Sario, Riemann surfaces, Princeton Univ. Press, Princeton, n. f, 1960.

2. W. S. Burnside, Theory of groups of finite order, Dover, 1911.

3. H. Florack, Reguläre und meromorphe Funktionen auf nicht geschlossenen Riemannschen Flächen, Schr. Math. Inst. Univ. Münster (1948), 34 pp.

4. H. Grauert, Analytische Faserungen über holomorph-vollständigen Räumen, Math. Ann. 135 (1958), 263-273.

5. M. Heins, Algebraic structure and conformal mapping, Trans. Amer. Math. Soc. 89 (1958), 267-276.

6. A. Hurwitz, Uber Riemannsche Flächen mit gegebenen Verzweigungspunkten, Math. Ann. 39 (1891), 1-61.

7. —, Über die Anzahl der Riemannschen Flächen mit gegebenen Verzweigungspunkten, Math. Ann. 55 (1902), 53-66.

8. H. J. Nastold, Uber meromorphe Schnitte komplex-analytischer Vektorraumbündel und Anwendungen auf Riemannsche Klassen. I, Math. Z. 69 (1958), 366-394.

9. O. Perron, Algebra. II, W. de Gruyter, Berlin, 1933.

10. K. Reidemeister, Fundamentalgruppe und Überlagerungsräume, Nachr. Ges. Wiss. Göttingen (1928), 69-76.

11. H. Röhrl, Das Riemann-Hilbertsche Problem der Theorie der linearen Differentialgleichungen, Math. Ann. 133 (1957), 1-25.

12. - On holomorphic families of fiber bundles over the Riemannian sphere, Mem. Coll. Sci. Kyoto 33 (1961), 435-477.

13. H. L. Royden, Rings of analytic and meromorphic functions, Trans. Amer. Math. Soc. 83 (1956), 269-276.

14. H. Seifert and W. Threlfall, Lehrbuch der Topologie, Teubner, Leipzig, 1934.

15. K. Stein, Analytische Zerlegungen komplexer Räume, Math. Ann. 132 (1956), 63-93.

16. H. Zassenhaus, Lehrbuch der Gruppentheorie, Hamburger Einzelschr., Leipzig, 1937.

UNIVERSITY OF MinNesota, INSTITUTE OF TeChNOLOGy, MinNEAPOLIS, MinNeSOTA 